\title{
Secondary breakup of axisymmetric liquid drops. I. Acceleration by a constant body force
}

\author{
Jaehoon Han and Grétar Tryggvason \\ Mechanical Engineering and Applied Mechanics, The University of Michigan, Ann Arbor, \\ Michigan 48109-2121
}

(Received 2 December 1998; accepted 16 August 1999)

\begin{abstract}
The secondary breakup of liquid drops, accelerated by a constant body force, is examined for small density differences between the drops and the surrounding fluid. Two cases are examined in detail: a density ratio close to unity $\left(\rho_{d} / \rho_{o}=1.15\right.$, where the Boussinesq approximation is valid) and a density ratio of ten. A finite difference/front tracking numerical technique is used to solve the unsteady Navier-Stokes equations for both the drops and the surrounding fluid. The breakup is controlled by the Eötvös number (Eo), the Ohnesorge number (Oh), and the viscosity and density ratios. If viscous effects are small (small Oh), the Eötvös number is the main controlling parameter. In the Boussinesq limit, as Eo increases the drops break up in a backward facing bag, transient breakup, and a forward facing bag mode. At a density ratio of ten, similar breakup modes are observed, with the exception that the forward facing bag mode is replaced by a shear breakup mode. Similar breakup modes have been seen experimentally for much larger density ratios. Although a backward facing bag is seen at low Oh, where viscous effects are small, comparisons with simulations of inviscid flows show that the bag breakup is a viscous phenomenon, due to boundary layer separation and the formation of a wake. At higher Oh, where viscous effects modify the evolution, the simulations show that the main effect of increasing Oh is to move the boundary between the different breakup modes to higher Eo. The results are summarized by "breakup maps" where the different breakup modes are shown in the Eo-Oh plane for different values of the viscosity and the density ratios. (C) 1999 American Institute of Physics. [S1070-6631(99)01112-5]
\end{abstract}

\section{INTRODUCTION}

In spray combustion, liquid atomization is a two stage process: In the primary breakup, a liquid jet emerging from the injector breaks up into drops which subsequently undergo secondary breakup into even smaller drops. Secondary breakup increases the total surface area of the fuel-air interface, thus enhancing the rate at which the fuel evaporates and burns.

Current computational models used for engineering predictions of spray combustion do not resolve the motion of individual drops. Instead, the effect of the drops is accounted for by subgrid models, computed in either an Eulerian or a Lagrangian way. For recent descriptions and reviews, see Drew and Passman ${ }^{1}$ and Crowe, Sommerfeld, and Tsuji. ${ }^{2}$ In Lagrangian models, the drops are represented by point particles, that can be split into two or more particles to represent drop breakup. For a description and application of breakup models in spray combustion simulations, see Reitz and Diwakar, ${ }^{3}$ O'Rourke and Amsden, ${ }^{4}$ Liu, Mather, and Reitz, ${ }^{5}$ Liu and Reitz, ${ }^{6}$ Kim and Wang, ${ }^{7}$ and Kong, Han, and Reitz. ${ }^{8}$ Two different approaches are typically used to model the breakup. The Taylor analogy breakup (TAB) model of O'Rourke and Amsden ${ }^{4}$ is based on an analogy between an oscillating and distorting liquid drop and a spring-mass system suggested by Taylor. ${ }^{9}$ The Reitz wave instability model, ${ }^{6}$ on the other hand, is based on a linear stability analysis for liquid jets. Both of these simplified models contain adjust- able parameters that must be determined by experimental studies.

In experiments, the drops are usually accelerated by a shock wave causing a step change in the velocity of the drop relative to the surrounding fluid, or by a constant body force such as gravity. The results are generally presented in terms of four nondimensional parameters: the relative strength of inertia and surface tension which is characterized by the Weber number for an impulsive acceleration and the Eötvös number for an acceleration by a constant body force; the ratio of viscous stresses and surface tension given by the Ohnesorge number; the density ratio; and the viscosity ratio of the drop and surrounding fluids.

Early experimental studies of drop breakup due to impulsive acceleration include those of Lane, ${ }^{10}$ who studied the shattering of liquid drops in steady or transient streams of air, and Hinze. ${ }^{11}$ The findings of Lane ${ }^{10}$ and Hinze ${ }^{11}$ have been extended to a broader range of parameters by Haas, ${ }^{12}$ Hanson, Domich, and Adams, ${ }^{13}$ Ranger and Nicholls, ${ }^{14}$ Gel'fand, Gubin, Kogarko, and Komar, ${ }^{15}$ Borisov, Gel'fand, Natanzon, and Kossov, ${ }^{16}$ and others. Krzeczkowski ${ }^{17}$ showed that the effect of drop viscosity is not significant when the Ohnesorge number based on the drop properties is less than about 0.1. Pilch and Erdman ${ }^{18}$ examined the drop size distribution for the so-called bag breakup mode and found that it was made up of a large number of small drops produced from the burst of the bag, and a few large drops originating from the annular rim. Wierzba ${ }^{19}$ reviewed the literature and 
found that there was a large variation in the reported values of the critical Weber numbers for the onset of the bag type breakup. His own experiments showed that small changes in the experimental conditions could affect the drop breakup significantly. Experimental investigations of the breakup of falling drops have typically been motivated by interest in the evolution of raindrops. For early experiments, see the study of the breakup of large drops by Magarvey and Taylor, ${ }^{20}$ for example. The secondary breakup of liquid drops due to both impulsive and continuous disturbances has been examined extensively by Hsiang and Faeth ${ }^{21-23}$ using a shock tube and drop towers. The majority of the data are for atmospheric conditions $\left(\rho_{d} / \rho_{o}>500, \mathrm{Re}>100, \mathrm{Oh}_{d}<0.1\right)$, although a limited number of studies for smaller density ratios and higher viscosity were also done. For the impulsive disturbance case, droplet deformation and breakup maps similar to those produced by Hinze ${ }^{11}$ and Krzeczkowski ${ }^{17}$ were constructed for a wide range of parameters. Joseph, Belanger, and Beavers ${ }^{24}$ studied the breakup of both Newtonian and non-Newtonian drops in a high-speed air stream. Their experiments, using a shock tube, resulted in a very high initial acceleration of drops and the authors stated that the Rayleigh-Taylor instability was the primary cause of breakup. For a more extensive review of experimental studies of secondary breakup of drops, see Clift, Grace, and Weber, ${ }^{25}$ Lefebvre, ${ }^{26}$ Bayvel and Orzechowski, ${ }^{27}$ and Sadhal, Ayyaswamy, and Chung. ${ }^{28}$

Most of the experimental studies mentioned previously are concerned with the breakup of liquid drops in air due to impulsive accelerations. The density and viscosity ratios are much higher than those considered in the present study. While those experimental results are not directly comparable to our simulations, the major breakup modes remain similar. We therefore summarize the major results of experimental studies of impulsively accelerated drops here. When the Ohnesorge number is small, the effects of drop viscosity can be neglected. At low We, the drops deform but do not break up. As the acceleration increases past a critical value, the drops become progressively flatter and eventually break up. As the Weber number is increased, four well defined breakup modes are observed (see, for example, Nigmatulin ${ }^{29}$ ).

(1) Vibrational breakup mode where the drop disintegrates into two or four equal-sized smaller drops.

(2) Bag breakup mode where the original drop deforms into a torus-shaped rim spanned by a thin fluid film that ruptures into tiny droplets, followed by disintegration of the rim into larger droplets.

(3) Shear breakup mode where small drops are continuously stripped off the rim of the original drop.

(4) Explosive breakup mode where strong surface waves disintegrate the drop in a violent manner.

This categorization and terminology are somewhat arbitrary, and other variations have been suggested by other researchers. For example, mode 3 has also been called "stripping-type breakup.", ${ }^{18,19}$ For low viscosity drops where the transition process shows no significant dependencies on $\mathrm{Oh}_{d}$, the critical Weber number is approximately 10 for the first transition, 20-60 for the second, and 1000 for the third. These numbers should be considered only as a rough guide because there are large variations in the critical Weber numbers in the available experimental data due to different test conditions. At higher $\mathrm{Oh}_{d}$, the We required for the onset of deformation and breakup increases with increasing $\mathrm{Oh}_{d}$.

Other researchers have examined the evolution of liquid drops in another liquid with a density comparable to the drop density, moving due to gravity. The deformation of miscible liquid drops at low Reynolds numbers was studied by Kojima, Hinch, and Acrivos, ${ }^{30}$ who observed that the drops form vortex rings. The stability of drops moving in immiscible fluids was investigated by Koh and Leal, $, 31,32$ who showed computational results for zero Reynolds number using a boundary integral method and experimental results for low Reynolds numbers. A similar investigation of the instability of drops, also using a boundary integral method was reported by Pozrikidis. ${ }^{33}$ Experiments by Baumann, Joseph, Mohr, and Renardy ${ }^{34}$ showed that vortex rings can also be created in immiscible liquids.

A few investigators have simulated the deformation and breakup of liquid drops numerically. However, due to the difficulties in dealing with large deformation of the interface and in accurately including surface tension along with viscous and inertial forces, such numerical simulations have often been based on considerable simplifications. The steady motion of deformable axisymmetric drops was investigated by Dandy and Leal ${ }^{35}$ at several Reynolds and Weber numbers using a finite-difference method. The steady rise of an axisymmetric drop in an unbounded surrounding fluid was examined by Volkov ${ }^{36}$ for intermediate Reynolds numbers. Bozzi, Feng, Scott, and Pearlstein ${ }^{37}$ presented finite-element simulations of the steady motion of axisymmetric drops in bounded domains. Fritts, Fyre, and $\operatorname{Oran}^{38}$ used a twodimensional Lagrangian finite-difference method to simulate the breakup of fuel droplets and Liang, Eastes, and Gharakhari $^{39}$ presented simulations of axisymmetric drop breakup using a Volume-of-Fluid method for a limited number of cases. Other numerical studies of the deformation and breakup of two-dimensional drops can be found in Deng and Jeng, ${ }^{40}$ Deng, Liaw, and Chou, ${ }^{41}$ Seung, Chen, and Chen, ${ }^{42}$ and Zaleski, Li, and Succi. ${ }^{43}$ However, these numerical results are still preliminary.

In spite of the progress made by previous investigators, several aspects of the secondary breakup are still not well understood, including the breakup of drops at high pressure and temperature, where experimental difficulties are encountered. It is also necessary to more closely examine the timedependent characteristics of the breakup. In existing spray models, the drop breakup is considered to occur instantaneously. Recent experimental evidence indicates, however, that secondary breakup takes finite time. Therefore, it is possible that the drop breakup should be treated as a timedependent process.

In this paper, a numerical method based on a front tracking technique that can accommodate large deformation of the drops is developed to simulate the breakup of liquid drops accelerated by a constant body force. The governing equations for axisymmetric geometry are solved numerically on a 


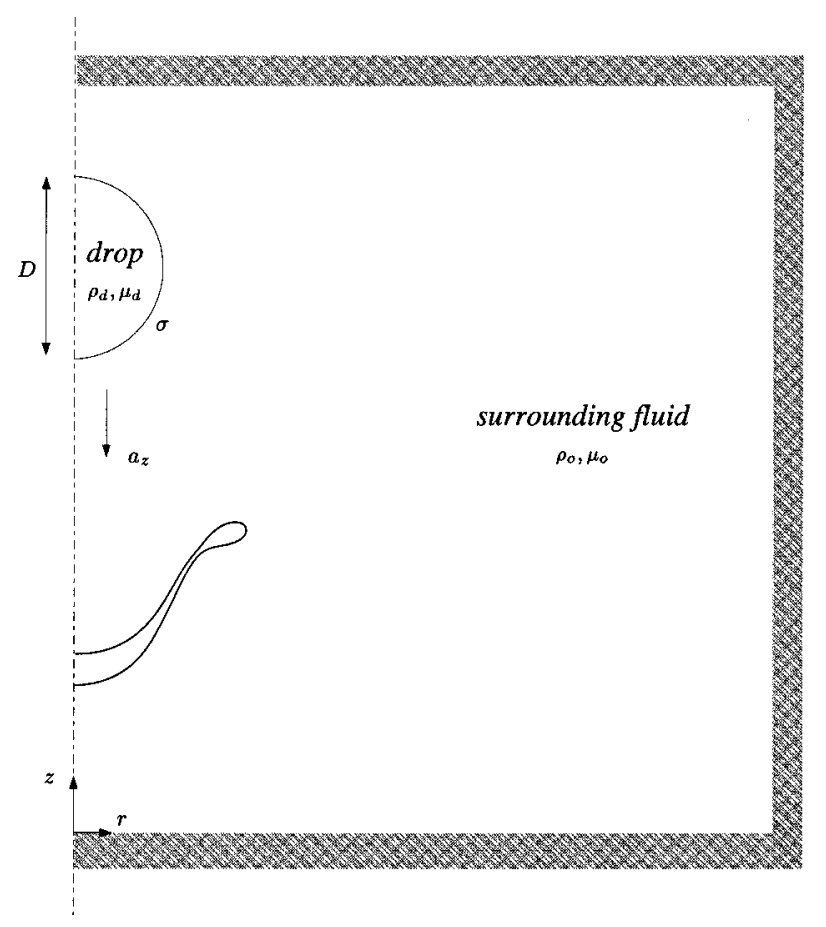

FIG. 1. Schematic illustration of the computational setup.

nonuniform grid using a finite-difference method. The drop interface is represented by connected marker points, whose positions are updated explicitly at each time-step. Results for a wide range of parameters are presented, and the physical significance of the results is discussed.

\section{FORMULATION AND NUMERICAL METHOD}

The physical problem and computational domain are sketched in Fig. 1; the left boundary is the axis of symmetry. We follow the motion of the fluids both inside and outside the drop and write a single set of equations for the whole flow field, using the conservative form of the governing equations to allow the density and viscosity to change discontinuously. Surface tension is added as a delta function to provide the proper interface boundary conditions. Written for an axisymmetric coordinate system, the Navier-Stokes equations are

$$
\begin{aligned}
\frac{\partial \rho u}{\partial t}+ & \frac{1}{r} \frac{\partial r \rho u^{2}}{\partial r}+\frac{\partial \rho \mathrm{v} u}{\partial z} \\
= & -\frac{\partial p}{\partial r}+\frac{\partial}{\partial r}\left(2 \mu \frac{\partial u}{\partial r}\right)+2 \mu \frac{\partial}{\partial r}\left(\frac{u}{r}\right)+\frac{\partial}{\partial z} \mu\left(\frac{\partial \mathrm{v}}{\partial r}+\frac{\partial u}{\partial z}\right) \\
& -\int_{S} \sigma \kappa \mathbf{n} \delta\left(\mathbf{x}-\mathbf{x}_{f}\right) d S \cdot \hat{i}_{r},
\end{aligned}
$$

$$
\begin{aligned}
\frac{\partial \rho \mathrm{V}}{\partial t}+ & \frac{1}{r} \frac{\partial r \rho u \mathrm{~V}}{\partial r}+\frac{\partial \rho \mathrm{V}^{2}}{\partial z} \\
= & -\frac{\partial p}{\partial z}+\frac{1}{r} \frac{\partial}{\partial r} \mu r\left(\frac{\partial \mathrm{v}}{\partial r}+\frac{\partial u}{\partial z}\right)+\frac{\partial}{\partial z}\left(2 \mu \frac{\partial \mathrm{v}}{\partial z}\right) \\
& -\int_{S} \sigma \kappa \mathbf{n} \delta\left(\mathbf{x}-\mathbf{x}_{f}\right) d S \cdot \hat{i}_{z}-\rho a_{z} .
\end{aligned}
$$

Here, $u$ and $\mathrm{v}$ are the velocity components in the radial and axial directions, $p$ is the pressure, and $\rho$ and $\mu$ are the discontinuous density and viscosity fields, $\sigma$ is the surface tension, $\kappa$ is twice the mean curvature, $\hat{i}_{r}$ and $\hat{i}_{z}$ are the radial and axial components of the surface unit normal vector pointing outward from the drop, and $\delta$ is a three-dimensional delta function. In (1) and (2), the surface tension is treated as a body force. The integral over the surface of the drop, $S$, results in a force that is smooth and continuous along the drop surface. In the numerical method, the delta function, $\delta$, is approximated by a smooth function with a compact but finite support. The constant acceleration gives rise to a body force in the axial direction denoted by $\rho a_{z}$.

The above equations are supplemented by the incompressibility condition

$$
\frac{1}{r} \frac{\partial r u}{\partial r}+\frac{\partial \mathrm{v}}{\partial z}=0
$$

which, when combined with the momentum equations, leads to an elliptic equation for the pressure

$$
\boldsymbol{\nabla} \cdot\left(\frac{\boldsymbol{\nabla} p}{\rho}\right)=\frac{1}{\rho} R,
$$

where $R$ is the divergence of the vector form of the momentum equations (1) and (2), excluding the pressure term.

We also have equations of state for the physical properties of the drop and the surrounding fluid

$$
\frac{D \rho}{D t}=0 ; \quad \frac{D \mu}{D t}=0
$$

where $D / D t$ is the material derivative. These two equations state that the physical properties of each fluid remain constant.

The numerical technique used for the simulations presented in this paper is based on the front-tracking/finitedifference method discussed in Unverdi and Tryggvason. ${ }^{44}$ The code employed in the present study is an axisymmetric version of the method. Since the axisymmetric code runs much faster than the fully three-dimensional version, it allows more runs and higher resolution. To improve the efficiency of the computations, the method was implemented on stretched grids to allow clustering of grid points in specific regions.

The momentum equations and the continuity equation are discretized using an explicit second-order predictorcorrector time-integration method and a second-order centered difference approximation for the spatial derivatives. The discretized equations are solved on a fixed, staggered 
grid using the Marker-and-Cell method developed by Harlow and Welch. ${ }^{45}$ The full-slip boundary condition is applied to all four boundaries.

To maintain a well defined boundary between the drops and the surrounding fluid, the boundary is marked by connected points (the front) that are advected by the fluid velocity, interpolated from the fixed grid. The new position of the marker points is used to construct a new density field by distributing the density jump to the grid points next to the front using area weighting, and integrating the jump to find the density everywhere. Once the density is known, the viscosity is set as a function of the density. The marker points are also used to find the surface tension, which is then assigned to the nearest grid points in the same way as the density jump, and added to the discrete Navier-Stokes equations. For a more detailed description of the front-tracking method, see Unverdi and Tryggvason ${ }^{44}$ and Tryggvason, Bunner, Ebrat, and Tauber. ${ }^{46}$

The implementation of the numerical technique to the drop breakup problem is straightforward and the method works well for a broad range of parameters. However, as $\rho_{d} / \rho_{o}$ is raised, the computational cost increases, partly because of the appearance of the coefficient $1 / \rho$ in the pressure equation (4) but also because the effect of the surrounding fluid is weaker when the density ratio is large and the drop travels a longer distance before breaking up. In order to avoid having to use a very long computational domain, we move the computational domain with the drop. The motion of the domain is determined from the solution, and an extra acceleration term is added to the governing equations to account for the time-dependent motion of the domain. The boundary conditions have also been modified to include a constant inflow at the bottom and a zero velocity gradient in the normal direction at the top.

The majority of the simulations presented here were carried out on HP 9000 workstations. A typical run required between 4000 and 120000 timesteps and took 12-240 hours, depending on the parameters of the problem.

Dimensional analysis shows that four independent dimensionless parameters govern the dynamics of drop deformation and breakup. When the drop is subject to an acceleration by a constant body force, it is convenient to use the Eötvös number, Eo (interchangeably called the Bond number, Bo) and the Ohnesorge number of the drop, $\mathrm{Oh}_{d}$, defined as

$$
\begin{gathered}
\mathrm{Eo}=\frac{a_{z} \Delta \rho D^{2}}{\sigma}, \\
\mathrm{Oh}_{d}=\frac{\mu_{d}}{\sqrt{\rho_{d} D \sigma}},
\end{gathered}
$$

where $\Delta \rho$ is the density difference between the drop and the surrounding fluid and $D$ is the initial diameter of the drop. The density and the viscosity ratios,

$$
\frac{\rho_{d}}{\rho_{o}} ; \quad \frac{\mu_{d}}{\mu_{o}},
$$

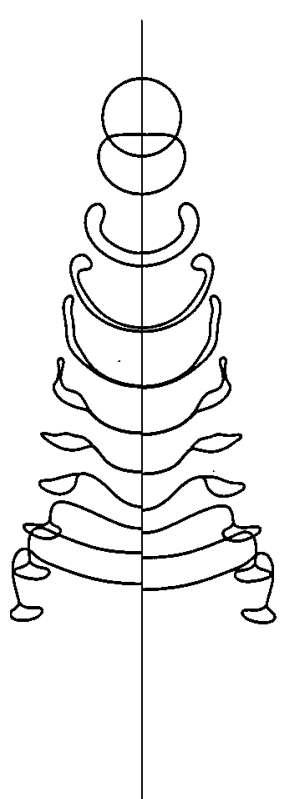

FIG. 2. Resolution test. The breakup of a drop computed using a 256 $\times 512$ grid (left) and a $512 \times 1024$ grid (right). $\rho_{d} / \rho_{o}=1.15$, Eo $=144$, $\mathrm{Oh}_{o}=0.05, \mathrm{Oh}_{d}=0.0466$. The drop shape is plotted every $\Delta t_{p}^{*}=3.873$.

can be selected as the other two parameters. The viscosity ratio is sometimes replaced by the Ohnesorge number based on the properties of the surrounding fluid

$$
\mathrm{Oh}_{o}=\frac{\mu_{o}}{\sqrt{\rho_{o} D \sigma}} .
$$

The subscripts, $d$ and $o$, denote the properties of the drop and the surrounding fluid, respectively. Time is nondimensionalized with respect to the drop diameter and the acceleration

$$
t^{*}=\frac{t}{\sqrt{D / a_{z}}} .
$$

\section{RESULTS AND DISCUSSION}

Numerical simulations are presented first for $\rho_{d} / \rho_{o}$ $=10$, using a moving computational domain. To examine the effect of the density ratio, simulations are also carried out for a small density ratio, $\rho_{d} / \rho_{o}=1.15$, using a fixed computational domain. For each density ratio, the effects of varying the other dimensionless parameters, $\mathrm{Eo}, \mathrm{Oh}_{o}$, and $\mathrm{Oh}_{d}$, are studied.

\section{A. Validation}

In order to validate the numerical method, grid refinement tests were performed. Typical results are presented in Fig. 2 where the shape of the drop is plotted at time intervals $\Delta t_{p}^{*}=3.873$, using two different grids: $256 \times 512$ (left) and $512 \times 1024$ (right). The nondimensional parameters are $\rho_{d} / \rho_{o}=1.15, \mathrm{Eo}=144, \mathrm{Oh}_{o}=0.05$, and $\mathrm{Oh}_{d}=0.0466$. Initially $\left(t^{*}=0\right)$, the drops are spherical and the velocities are zero everywhere. Despite the large deformation of the drop, the results agree well. In Fig. 3, the aspect ratio and the centroid velocity are plotted versus nondimensional time. 


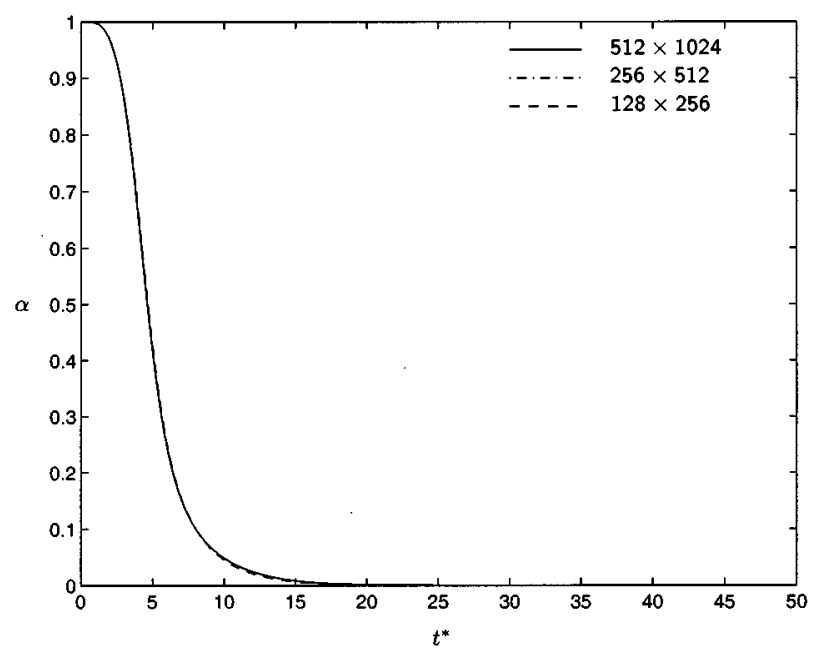

(a) aspect ratio

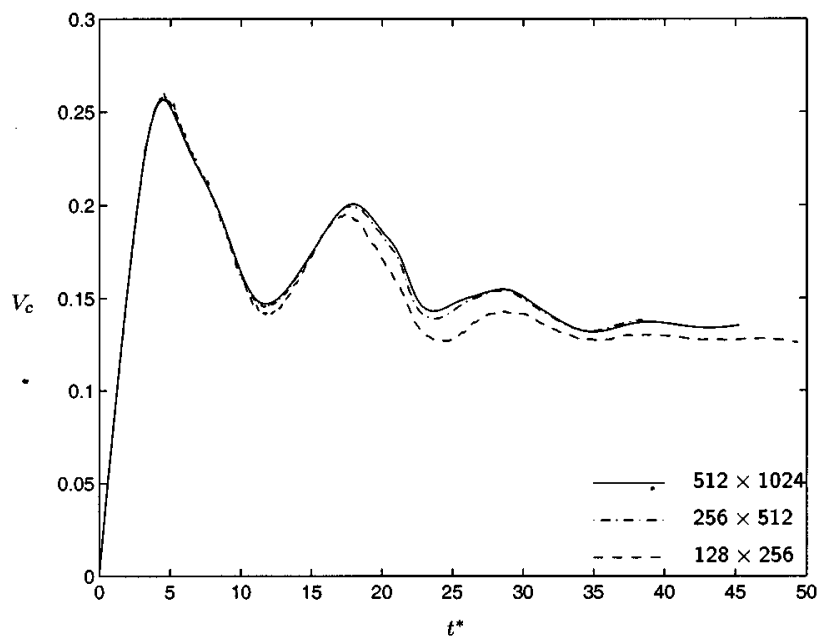

(b) centroid velocity

FIG. 3. Resolution test. Aspect ratio and centroid velocity plotted versus $t^{*}$. Results using three different grids, $128 \times 256,256 \times 512$, and $512 \times 1024$, are shown. $\rho_{d} / \rho_{o}=1.15, \mathrm{Eo}=144, \mathrm{Oh}_{o}=0.05, \mathrm{Oh}_{d}=0.0466$.

The aspect ratio is defined as the drop thickness at the centerline divided by the maximum width of the drop. The centroid velocity is found by taking the volume average of the vertical velocity inside the drop. The results corresponding to Fig. 2 are shown along with results using a coarser grid: $128 \times 256$. The result from the $128 \times 256$ grid shows a small difference but the two finer grids give nearly identical results.

In addition, we have compared our results to the steady state results for a single axisymmetric deformable drop computed by Dandy and Leal. ${ }^{35}$ They specified the Reynolds number and the Weber number and found the drag coefficient, $C_{d}$, as a part of the solution. In our transient simulation, it is not possible to specify Re and We a priori, since the velocity of the drop is computed as part of the solution. However, once the drag coefficient is known, the Eötvös number and Ohnesorge number can be found by

$$
\mathrm{Eo}=\frac{3}{4} \mathrm{We} C_{d} ; \mathrm{Oh}_{o}=\sqrt{\frac{\mathrm{We}}{\mathrm{Re}^{2}}}
$$

For a drop translating at a Reynolds number equal to 100 and Weber number equal to 4 , with $\rho_{d} / \rho_{o}=0.91$ and $\mu_{d} / \mu_{o}$ $=1$, Dandy and Leal ${ }^{35}$ found $C_{d}=0.919$ in an unbounded domain. This gives $\mathrm{Eo}=2.75$ and $\mathrm{Oh}_{o}=0.02$. Our computation was done using a $256 \times 768$ grid in a domain 5 and 15 times the initial diameter of the drop in the radial and axial directions, respectively. The computed Reynolds and Weber numbers differ from those given by Dandy and Leal ${ }^{35}$ by less than $1 \%$ when the drop reaches a steady state. For $\mu_{d} / \mu_{o}$ $=4$ and the same Re, We, $\rho_{d} / \rho_{o}$ as in the previous case, Dandy and Leal ${ }^{35}$ found $C_{d}=1.10$. Our computation was done using $\mathrm{Eo}=3.3, \mathrm{Oh}_{o}=0.02$, and the same $\rho_{d} / \rho_{o}$ and $\mu_{d} / \mu_{o}$, with the same resolution and domain size as in the previous case. The result gives $C_{d}=1.13$, which is approximately $3 \%$ higher. This is due to the finite size of our computational domain. Computations using domains of half and twice the original size in the radial direction yield $C_{d}$ $=1.19$ and 1.12 , respectively.

\section{B. The Boussinesq approximation}

Before presenting further computational results, we pause to examine the validity of the Boussinesq approximation. The Boussinesq approximation states that if the density difference is small, density variations are only important when multiplied by gravity. $\Delta \rho$ is therefore no longer an independent parameter and it is sufficient to simulate the breakup for only one value of the density ratio in this limit. Results for other values of $\Delta \rho$ can be obtained by simply rescaling time. For a discussion of the Boussinesq approximation to stratified flows, see, for example, Dahm, Scheil, and Tryggvason. ${ }^{47}$ The relative magnitude of the density difference is better expressed by the Atwood number, defined by

$$
A=\frac{\rho_{d}-\rho_{o}}{\rho_{d}+\rho_{o}} .
$$

When $A$ is sufficiently small $\left(\rho_{d} / \rho_{o} \rightarrow 1\right)$, time and velocities can be scaled by the average static pressure to yield

$$
\begin{aligned}
& \hat{t}=\frac{t}{\sqrt{D /\left(A a_{z}\right)}}, \\
& \hat{u}=\frac{u}{\sqrt{A a_{z} D}} ; \quad \hat{\mathrm{v}}=\frac{\mathrm{v}}{\sqrt{A a_{z} D}} .
\end{aligned}
$$

The Eötövs number and the Ohnesorge number must also be redefined as

$$
\begin{aligned}
& \widehat{\mathrm{EO}}=\frac{\rho_{a \mathrm{v}} A a_{z} D^{2}}{\sigma}, \\
& \widehat{\mathrm{Oh}}_{o}=\frac{\mu_{o}}{\sqrt{\rho_{a \mathrm{v}} D \sigma}},
\end{aligned}
$$

where $\rho_{a \mathrm{v}}=0.5\left(\rho_{d}+\rho_{o}\right)$. Note that the constant acceleration appears only as $A a_{z}$ instead of $a_{z}$ alone. 


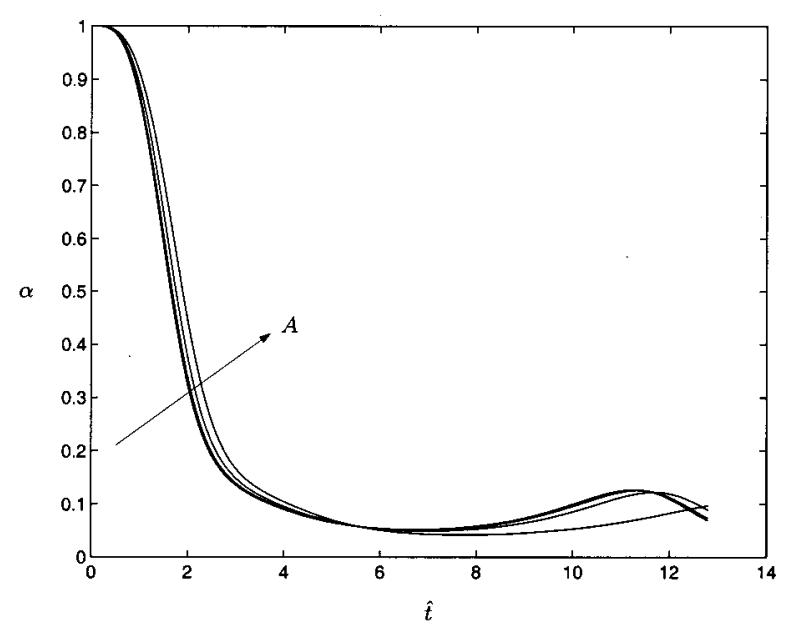

(a) aspect ratio

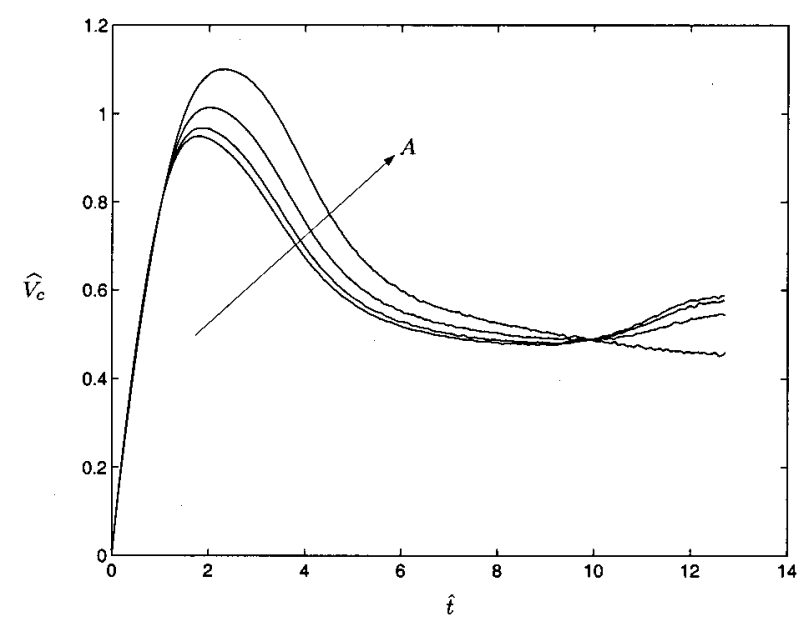

(b) centroid velocity

FIG. 4. Test of the Boussinesq approximation. Aspect ratio and centroid velocity plotted versus $\hat{t}$. Results are shown for four different Atwood numbers: $0.07,0.11,0.2$, and 0.33 . The corresponding density ratios are 1.15 , $1.25,1.5$, and 2.0. $\widehat{\mathrm{EO}}=72, \widehat{\mathrm{Oh}}_{o}=0.241$, and $\mu_{d} / \mu_{o}=1$.

In order to check the validity of the Boussinesq approximation, tests were done for different values of $A$. In Fig. 4, results for $\widehat{\mathrm{EO}}=72, \widehat{\mathrm{Oh}}_{o}=0.241$, and $\mu_{d} / \mu_{o}=1$ are presented. The computation was done using a $256 \times 768$ grid and the size of the computational domain was 5 and 15 times the initial drop diameter in the radial and the axial directions, respectively. The aspect ratio $\alpha$ and the nondimensionalized centroid velocity $\hat{V}_{c}$ are plotted versus $\hat{t}$ in (a) and (b), respectively. In each graph, simulations using four values of $A$ are shown: $0.07,0.11,0.2$, and 0.33 , corresponding to the density ratios: $1.15,1.25,1.5$, and 2.0 . The plots confirm that the scaling works well when $A$ is less than about 0.2 to 0.3 . The ability to cover this density range by a single simulation is obviously a considerable simplification.

\section{Effect of Eo at small Oh}

When $\mathrm{Oh}$ is small and surface tension is much more important than viscous stresses, Oh has little influence on the breakup and Eo is the only controlling parameter. Here, we present results for different Eo when $\mathrm{Oh}$ is small. When a drop is set into motion by a constant body force, the hydrodynamic pressure is higher at the poles and lower at the equator and the drop deforms into an oblate ellipsoid. This deformation is opposed by the surface tension. Depending on the relative strength of the pressure forces and the surface tension, measured by Eo, different breakup modes are observed.

In Fig. 5, the effect of Eo is presented for $\rho_{d} / \rho_{o}=10$ and $\mathrm{Oh}_{o}=\mathrm{Oh}_{d}=0.05$. The simulations are done using a moving coordinate system where the origin is fixed at the centroid of the drop. The domain has dimensions of five and fifteen times the initial drop diameter in the radial and axial directions, respectively. The centroid of the drop is fixed at a position five times the initial drop diameter above the bottom boundary. The evolution of the drop is shown for nine different values of Eo [(a) to (i)]. In each column, the drop interface is plotted at fixed time intervals. The separation between two successive drops is equal to the distance that the drop travels during the time interval.

In (a), the drop is shown for Eo=12. As the drop starts falling, the back side becomes flat while the front side retains a rounded shape. After the initial deformation, the drop reaches a steady state and no further change in the drop shape is seen. When Eo is increased to 24 in (b), the drop deformation is more pronounced. Initially, the drop assumes a shape similar to that shown in (a), but then the back of the drop becomes increasingly more convex and eventually the drop deforms into a thin disk-like shape that moves at a nearly steady state. The drop shown in (c) for Eo $=28.8$ evolves in the same way until it has deformed into a disk-like shape. Then the thickness of the drop near the symmetry axis continues to decrease, and most of the drop fluid moves outward toward the edge of the drop. Finally, the center of the front surface is pushed upward, forming a backward-facing bag. At this stage, most of the drop fluid is contained in the annular-shaped rim. As time progresses, the bag expands both radially outward and vertically upward. Experimental evidence indicates that the drop will eventually break into small drops. The evolution shown in (d) for Eo=36 is very similar to that in (c), displaying a backward-facing bag. The only difference is that the rate of deformation is higher and the backward-facing bag expands more rapidly.

When Eo is further increased to 48 in (e), a different mode of breakup is observed. The initial deformation is not very different from the previous cases, and an indentation develops on the back surface, but instead of deforming into a disk-like shape, the drop remains relatively thick near the symmetry axis and the edge of the drop is swept back in the downstream direction. A large wave then develops on the drop interface and as this wave propagates, the drop deforms in an erratic manner. The evolution of the drop shown in (f) for Eo $=60$ reveals another mode of deformation. The initial evolution is similar to the previous cases, but the results are different at later times. As the indentation at the top progressively deepens, the drop does not deform into a thin disk-like shape. Instead, the edge of the drop is deflected in the downstream direction and drawn out into a thin film with a blob of 


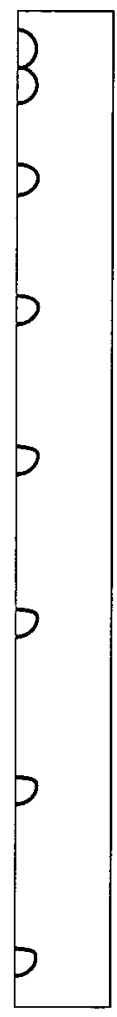

(a) $E o=12$

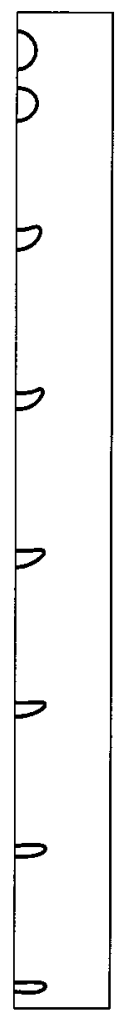

(b) $E o=24$

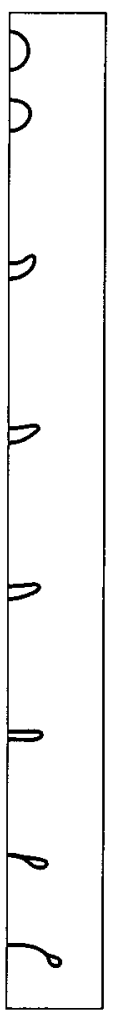

(c) $E_{0}=28.8$

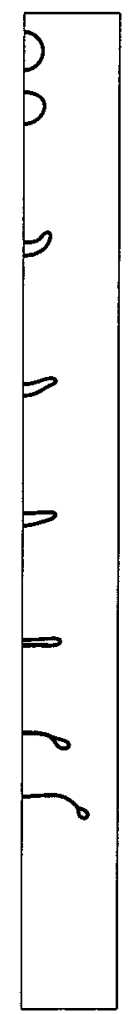

(d) $E o=36$

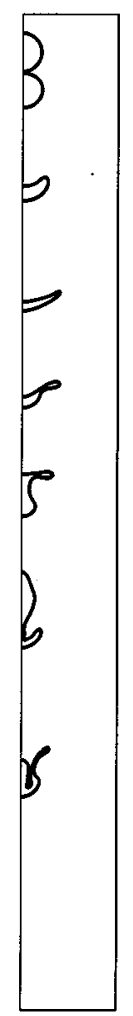

(e) $E o=48$

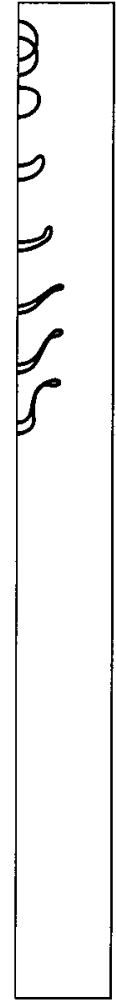

(f) $E o=60$

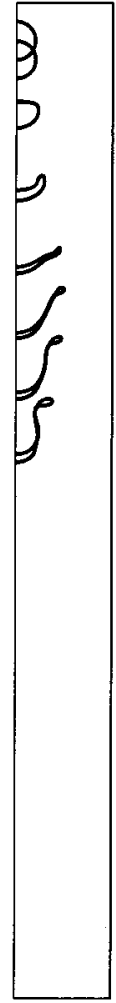

(g) $E o=72$

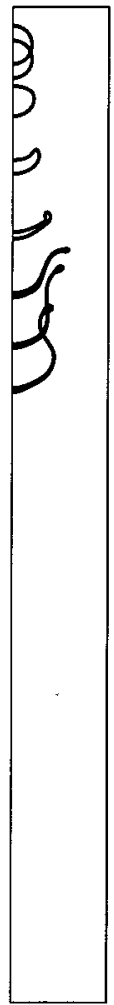

(h) $E o=96$

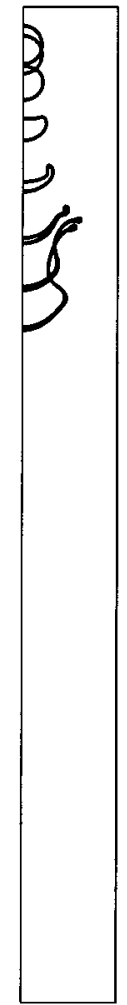

(i) $E o=144$

FIG. 5. Effect of Eo on the deformation of drops with $\rho_{d} / \rho_{o}=10 . \mathrm{Oh}_{o}=\mathrm{Oh}_{d}=0.05$. The simulations are done using a $256 \times 768$ grid for a moving computational domain of dimensions $5 \times 15$ times the initial drop diameter. The boundaries of the column do not indicate the actual boundaries of the computational domain. The gap between two successive drops in each column represents the distance the drop travels at a fixed time interval and the last interface is plotted at $t^{*}=$ (a) 11.19 ; (b) 15.82 ; (c) 14.85 ; (d) 13.83 ; (e) 11.19 ; (f) 7.15 ; (g) 7.83 ; (h) 6.78 ; (i) 5.54 .

drop fluid at the end. The appearance of this film is similar to the skirted drop shapes observed in experimental studies of liquid drops moving at a steady state (Wairegi and Grace ${ }^{48}$ ). The center portion of the drop, however, maintains a convex shape and its thickness at the symmetry axis stops decreasing. Similar drop deformations are observed at even higher $\mathrm{Eo}=72,96$, and 144 as shown in (g), (h), and (i), respectively.

Based on these results, the evolution of drops with $\rho_{d} / \rho_{o}=10$ at a small Oh can be classified into four categories in order of increasing Eo: steady deformation, formation of a backward-facing bag, transient breakup with a complex shape, and stripping or shearing of a film from the edge of the drop. It is evident from Fig. 5 that drops breaking up in the backward-facing mode travel a much longer distance than those breaking up in the shear breakup mode. Also note that for the same breakup mode, the rate of drop deformation increases as Eo increases.

In Fig. 6, the evolution of a drop with a small density ratio, $\rho_{d} / \rho_{o}=1.15$, is shown for different Eo. Again, values of Ohnesorge numbers, $\mathrm{Oh}_{o}=0.05$ and $\mathrm{Oh}_{d}=0.0466$ are chosen so that viscous stresses are small compared to surface tension. The computations were done using a fixed coordinate system. When Eo is small, the drop deforms into an oblate ellipsoid and moves with a steady state shape as shown in (a) for Eo=12. When Eo is increased to 24 (b), the drop deforms more and eventually forms a backward-facing bag as observed for $\rho_{d} / \rho_{o}=10$ in Fig. 5(c). In (c), where Eo is 48, the drop moves with an essentially steady convex shape, showing no sign of bag formation. Compared to its high density ratio counterpart shown in Fig. 5(e), the overall deformation is reduced. When Eo is 96 in (d), the indentation at the back of the drop deepens continuously until it reaches the front of the drop, creating a forward-facing bag. Eventually, however, the heavier edge falls faster than the thin bag. This formation of a forward-facing bag is different from the shear breakup mode observed in Figs. 5(f)-5(i), where a significant portion of the fluid remains near the symmetry axis while a thin film is pulled away from the edge. In (e), Eo is further increased to 144 . The overall evolution is similar to (d), but the rate of deformation is slightly faster.

In Fig. 7, vorticity contours (left) and streamlines with respect to a frame moving with the drop (right) are plotted at a few selected times for the drop shown in Fig. 5(c). Most of the vorticity is created at the outer edge of the drop, as expected, and the streamlines show the formation of a large wake behind the drop. The pressure difference between the front stagnation point and the wake causes the formation of the backward-facing bag.

Figure 8 shows vorticity contours (left) and streamlines (right) at a few selected times for the drop shown in Fig. 5(f). Although vorticity generated at the drop surface accumulates into a large wake as in Fig. 7, the more deformable drop is continuously deformed by the flow (as seen by streamlines crossing its boundary) and the edge is pulled back by the flow. In the last frame, the large wake formed initially sepa- 


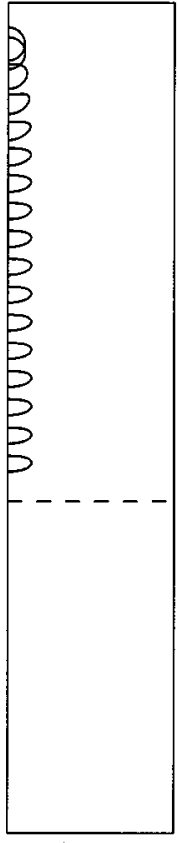

(a) $E o=12$

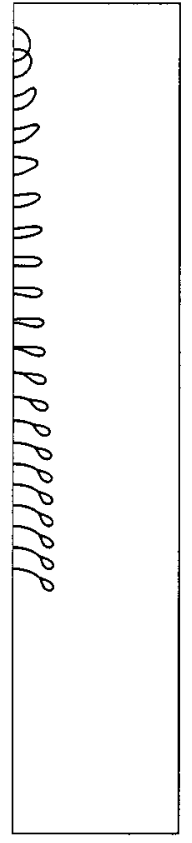

(b) $E o=24$

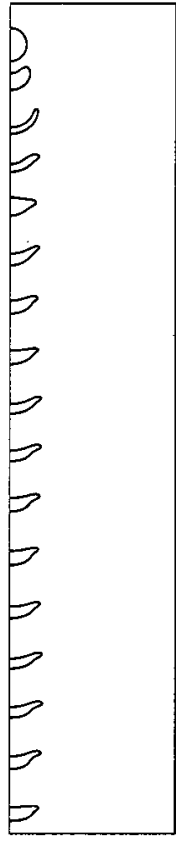

(c) $E o=48$

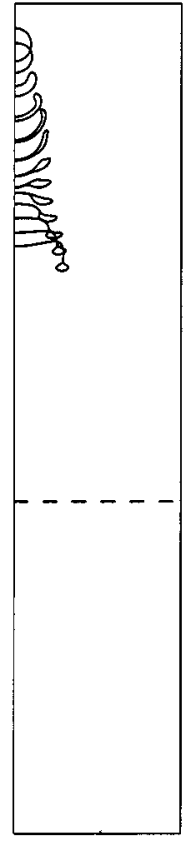

(d) $E o=96$

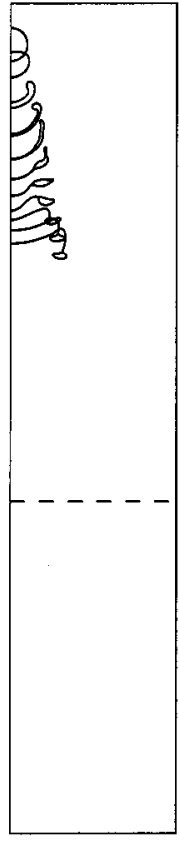

(e) $E o=144$

FIG. 6. Effect of Eo on the deformation of drop with $\rho_{d} / \rho_{o}=1.15 . \mathrm{Oh}_{o}=0.05, \mathrm{Oh}_{d}=0.0466$. The simulations are done using a $256 \times 1280$ grid in (b) and (c) and a $256 \times 768$ grid in (a), (d), and (e). The fixed computational domain has a dimension of $5 \times 15$ times the initial drop diameter in (b) and (c) and 5 $\times 25$ times the initial drop diameter in (a), (d), and (e). The dashed line in (a), (d), and (e) represents the actual bottom boundary of the computational domain. The gap between two successive drops in each column represents the distance the drop travels at a fixed time interval and the last interface is plotted at $t^{*}=$ (a) 44.72; (b) 79.06; (c) 89.44; (d) 37.94; (e) 38.73 .

rates from the drop, leaving the film to move toward the axis due to the flow around the smaller remaining drop.

In Fig. 9, vorticity contours (left) and streamlines (right) are shown for the drop in Fig. 6(e). Here, the vorticity generated at the interface moves with the drop, forming a dipole that continuously deforms the interface into a forward-facing bag.

Figures 10 and 11 show the centroid velocity of the drop $V_{c}$ plotted versus $t^{*}$ for the drops shown in Figs. 5 and 6 , respectively. Since the velocity is nondimensionalized by $\sqrt{a_{z} D}$, the graphs for different values of Eo all have the same initial slope. After the initial acceleration, the drop deformation, which depends on Eo, determines the velocity. In Fig. 10, drops with low Eo deform less and therefore move faster than drops with high Eo. The lowest Eo drop $(E o=12)$ asymptotically reaches a steady state velocity, but the other drops all slow down as they start deforming. The Eo=24

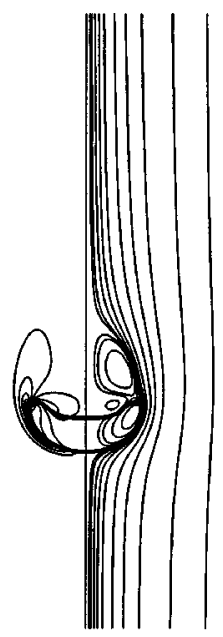

(a) $t^{*}=4.24$

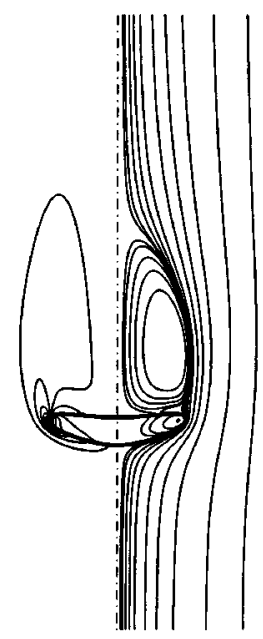

(b) $t^{*}=8.48$

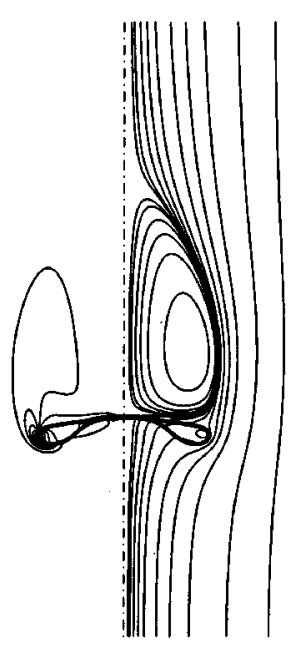

(c) $t^{*}=12.7$

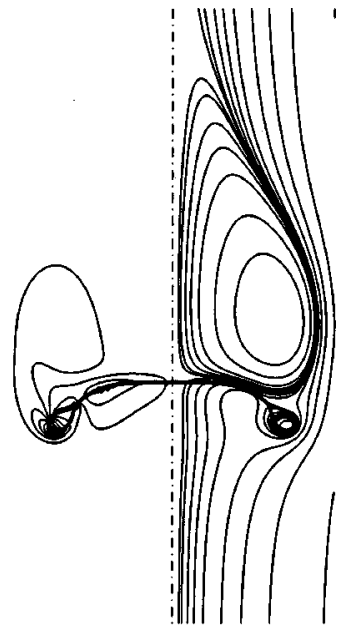

(d) $t^{*}=17.0$

FIG. 7. Vorticity contours (left) and streamlines (right) for the drop in Fig. 5(c). $\rho_{d} / \rho_{o}=10$, Eo=28.8, $\mathrm{Oh}_{o}=\mathrm{Oh}_{d}=0.05$. The results are shown for four selected times. 


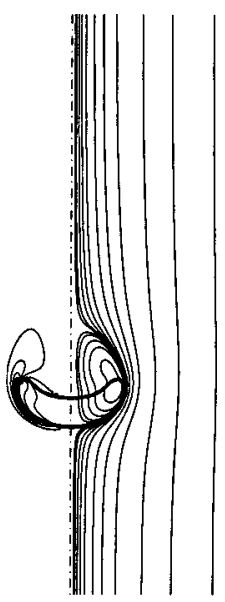

(a) $t^{*}=\mathbf{3 . 0 6}$

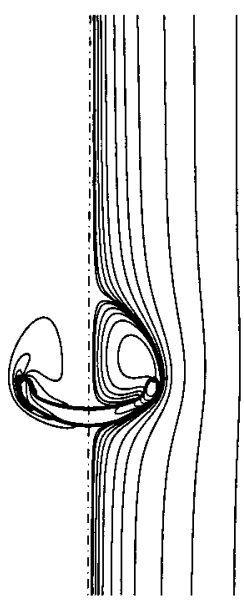

(b) $t^{*}=4.08$

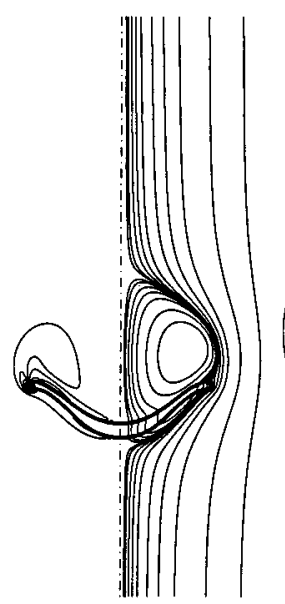

(c) $t^{*}=5.10$

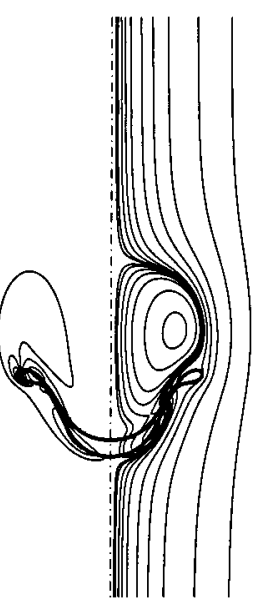

(d) $t^{*}=6.12$

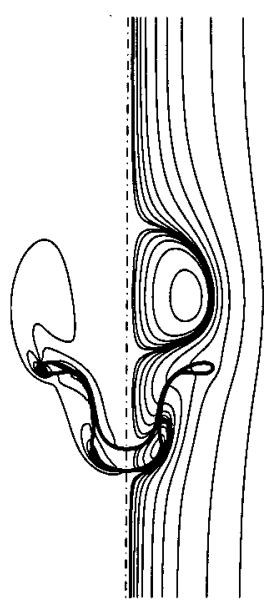

(e) $t^{*}=7.14$

FIG. 8. Vorticity contours (left) and streamlines (right) for the drop in Fig. 5(f). $\rho_{d} / \rho_{o}=10, \mathrm{Eo}=60, \mathrm{Oh}_{o}=\mathrm{Oh}_{d}=0.05$. The results are shown for five selected times.

drop also reaches a steady velocity. The drops that undergo bag breakup first behave like the Eo=24 drop, but as the bag forms, the drops slow down rapidly. At the very end, the $E 0=36$ drop speeds up again, as the rim of the drop starts falling independently of the bag. The rest of the drops all slow down rapidly as they are stretched perpendicular to the flow, and all speed up again as the thin film pulled from their edges folds back toward the axis. The results for the small density difference in Fig. 11 show a similar trend, but with a few differences. The transient drop $(\mathrm{Eo}=48)$ reaches a velocity that is nearly the same as the velocity of the drop moving with a steady deformed shape $(E \mathrm{E}=12)$ and the reduction in speed due to bag breakup is smaller than in Fig. 10.

In Figs. 12 and 13, the surface area $S$ (normalized by the initial value $S_{o}$ ) is plotted versus $t^{*}$ for the drops shown in Figs. 5 and 6, respectively. The graphs for $\rho_{d} / \rho_{o}=10$ in Fig. 12 , show that a rapid increase of the surface area takes place when breakup occurs, and that the backward-facing bag breakup mode takes longer than the shear breakup mode. The drops undergoing a shear breakup show a reduction in surface area when the film moves toward the symmetry axis. The graphs for $\rho_{d} / \rho_{o}=1.15$ in Fig. 13 also display a rapid increase of the surface area when the drops break up. However, the drops with the highest Eo show a rapid increase in surface area after the rim starts falling, and the surface area of the drop undergoing bag breakup grows relatively slowly compared to the higher density ratio drops.

\section{Effect of Oh}

Figure 14 illustrates the effect of the Ohnesorge number (the nondimensional viscosity) for drops with a finite density ratio, $\rho_{d} / \rho_{o}=10$. The drops are shown at several times. Here, $\mathrm{Oh}_{d}$ is equal to $\mathrm{Oh}_{o}$. The case where $\mathrm{Oh}_{d}$ is different from $\mathrm{Oh}_{o}$ will be discussed in the next section. In the top row

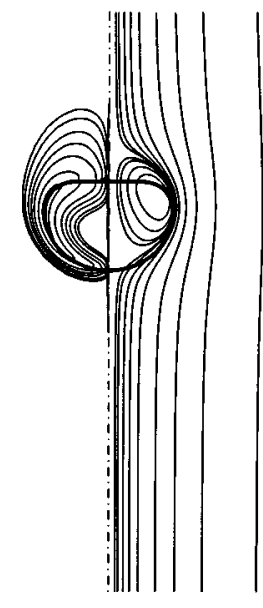

(a) $t^{*}=3.87$

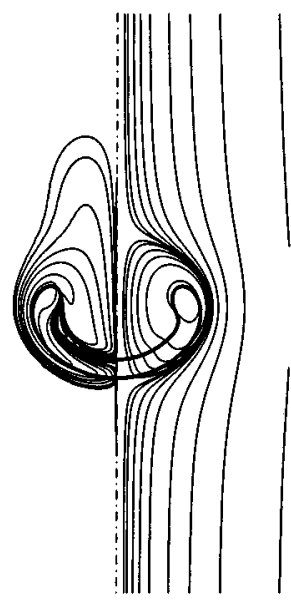

(b) $t^{*}=7.75$

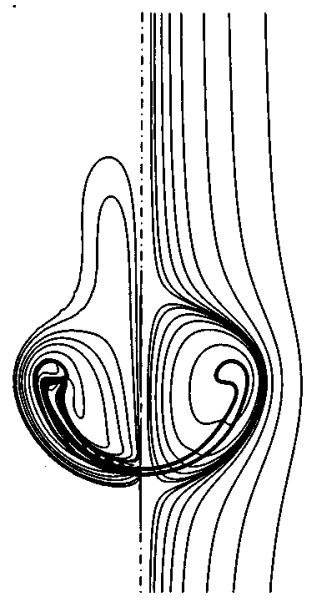

(c) $t^{*}=11.6$

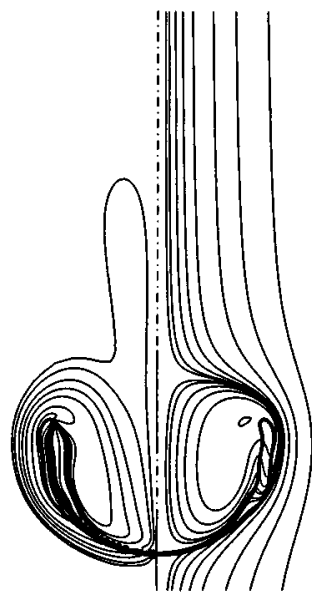

(d) $t^{*}=15.5$

FIG. 9. Vorticity contours (left) and streamlines (right) for the drop in Fig. 6(e). $\rho_{d} / \rho_{o}=1.15, \mathrm{Eo}=144, \mathrm{Oh}_{o}=0.05, \mathrm{Oh}_{d}=0.0466$. The results are shown for four selected times. 


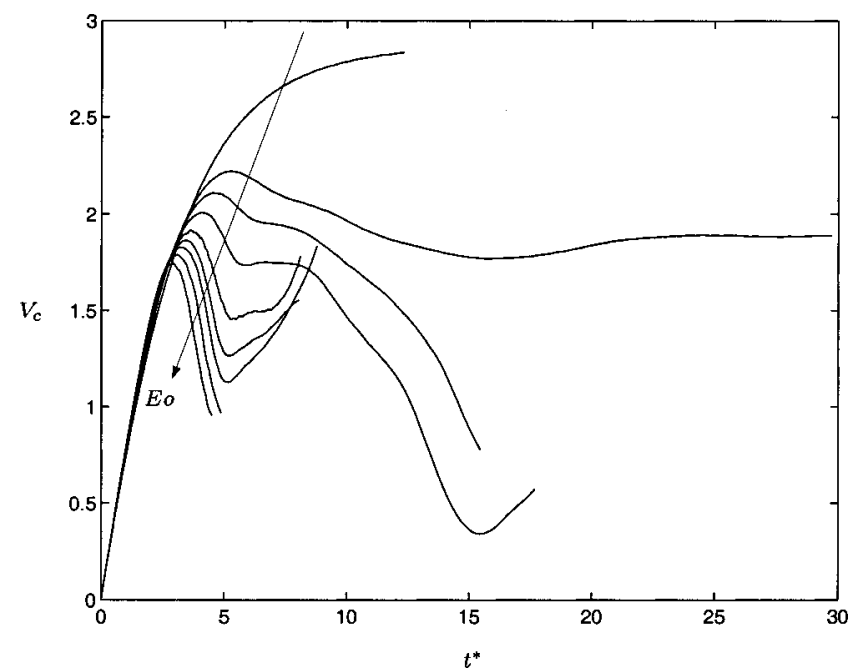

FIG. 10. Centroid velocity versus $t^{*}$ for the drops shown in Fig. 5. The results are presented for $\mathrm{Eo}=12,24,28.8,36,48,60,72,96$, and 144. $\rho_{d} / \rho_{o}=10, \mathrm{Oh}_{o}=\mathrm{Oh}_{d}=0.05$.

[(a)-(c)], Oh=0.05, 0.125, and 0.25, from left to right, and $\mathrm{Eo}=28.8$. The $\mathrm{Oh}=0.05$ case (a) has already been shown in Fig. 5(c), but is included here for comparison. The initial deformation of all three drops is similar, but whereas the $\mathrm{Oh}=0.05$ drop (a) deforms into a backward-facing bag, the other two drops reach a steady state shape. Of those, the less viscous drop (b) is flatter.

In the bottom row [(d)-(f)], Eo is increased to 144 and the evolution of the drops is presented for the same three values of $\mathrm{Oh}$ as in the top row. In (d), the drop already shown in Fig. 5(i) is included for reference. This drop undergoes a so-called shear (or boundary stripping) breakup. The $\mathrm{Oh}=0.125$ drop (e) shows a similar evolution as the drop in (d), although the rate of deformation is reduced slightly. The center portion of the drop still contains a significant amount of drop fluid and formation of a backward-facing bag, which requires the formation of a very thin film of fluid near the

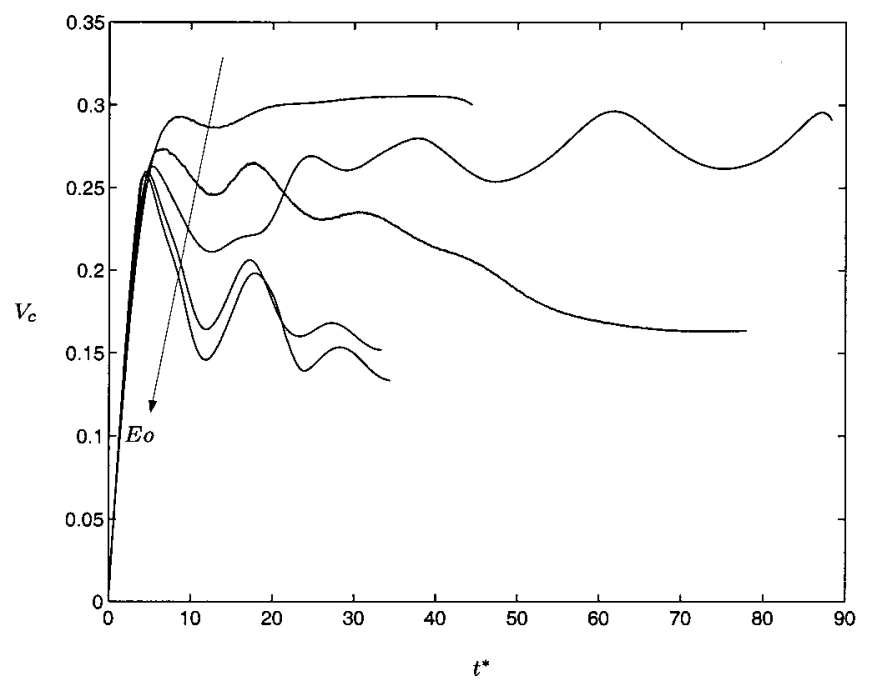

FIG. 11. Centroid velocity versus $t^{*}$ for the drops shown in Fig. 6. The results are presented for $\mathrm{Eo}=12,24,48,96$, and 144. $\rho_{d} / \rho_{o}=1.15, \mathrm{Oh}_{o}$ $=0.05, \mathrm{Oh}_{d}=0.0466$.

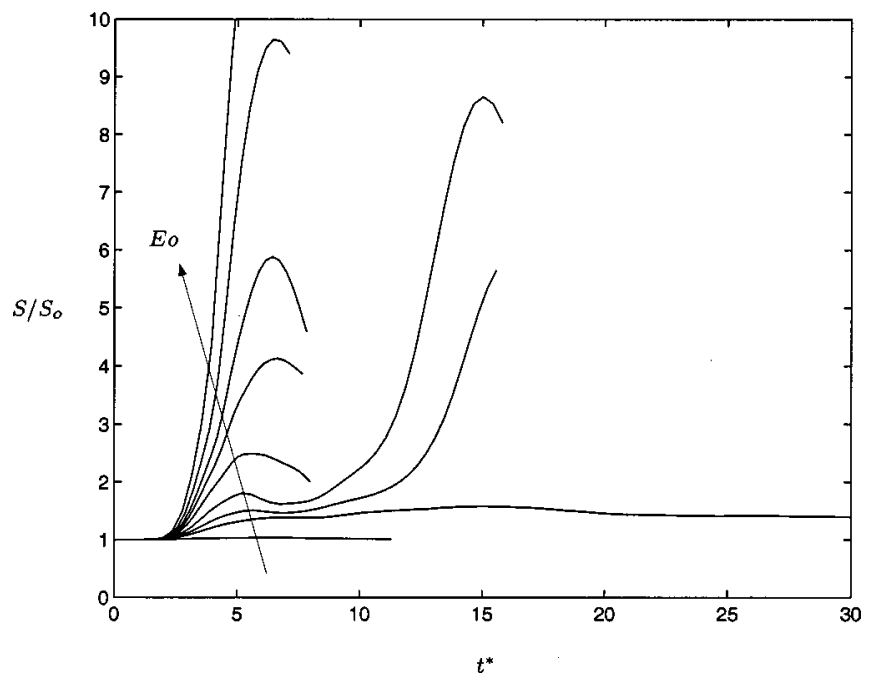

FIG. 12. Normalized surface area versus $t^{*}$ for the drops shown in Fig. 5. The results are presented for $E o=12,24,28.8,36,48,60,72,96$, and 144. $\rho_{d} / \rho_{o}=10, \mathrm{Oh}_{o}=\mathrm{Oh}_{d}=0.05$.

symmetry axis, does not occur. In contrast, the center portion of the drop in (f) is drained completely, and the drop forms a backward-facing bag.

Based on the results shown in Fig. 14, it is clear that increasing both $\mathrm{Oh}_{o}$ and $\mathrm{Oh}_{d}$ simultaneously results in a translation of the boundaries between the breakup modes to higher Eo.

Figure 15 illustrates the effect of viscosity on the initial deformation of drops with $\rho_{d} / \rho_{o}=1.15$. In addition to runs with a finite viscosity, we show simulations with zero viscosity, obtained by an axisymmetric vortex method (see Dahm, Frieler, and Tryggvason ${ }^{49}$ ). In the top row, Eo $=24$ and $\mathrm{Oh}_{o}$ is $0.05,0.025,0.01$, and 0 from left to right. In all cases, $\mu_{d} / \mu_{o}=1$. While the initial acceleration is dominant, all the drops evolve in the same way. As time progresses, viscosity effects become important and the viscous drops will eventually develop a backward-facing bag due to the

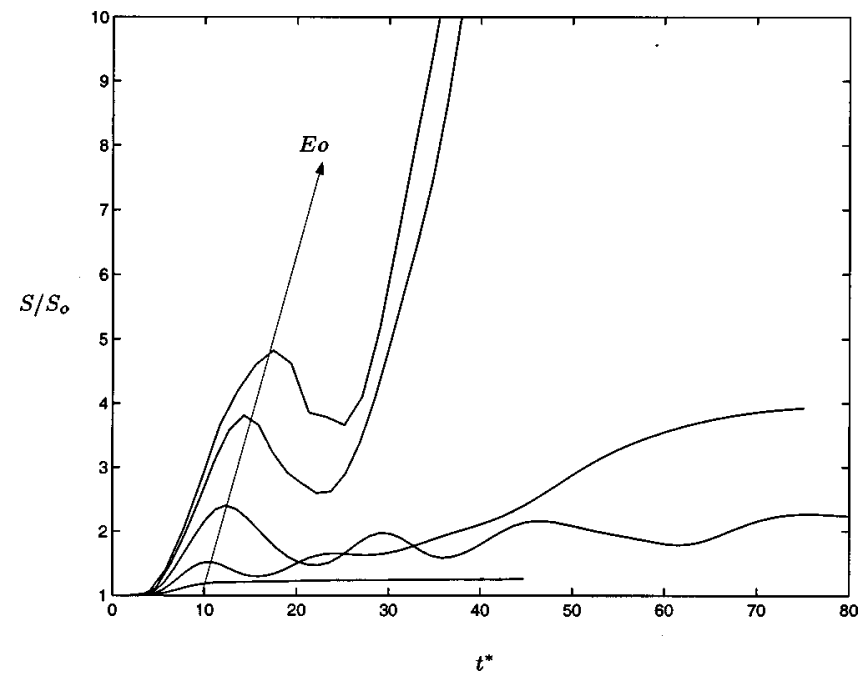

FIG. 13. Normalized surface area versus $t^{*}$ for the drops shown in Fig. 6 . The results are presented for $\mathrm{Eo}=12,24,48,96$, and 144. $\rho_{d} / \rho_{o}=1.15$, $\mathrm{Oh}_{o}=0.05, \mathrm{Oh}_{d}=0.0466$. 

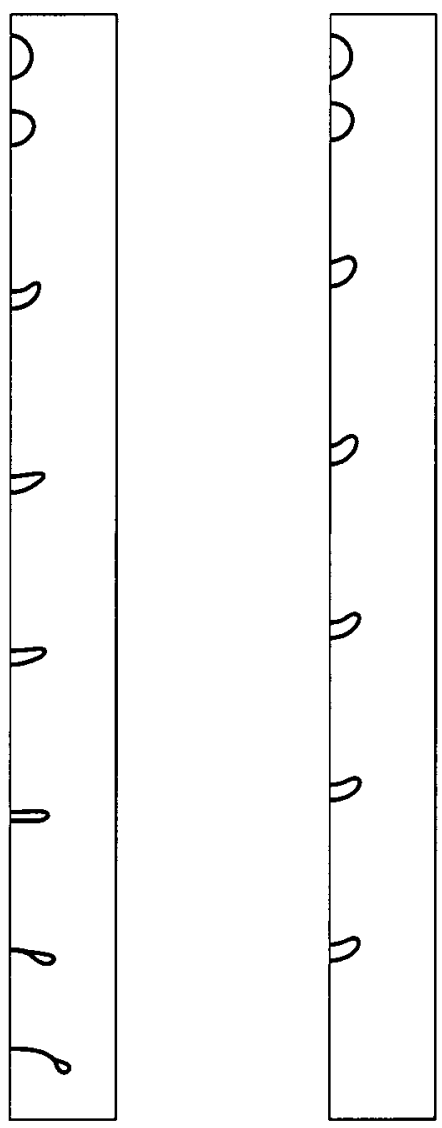

(a) $\mathrm{Oh}=0.05$

(b) $O h=0.125$

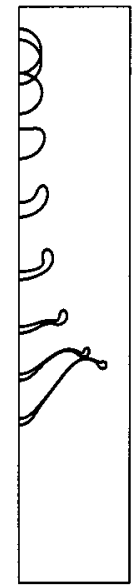

(d) $O h=0.05$

(e) $O h=0.125$

(f) $O h=0.25$

FIG. 14. Effect of Oh for drops with $\rho_{d} / \rho_{o}=10$. The drop evolution is shown for three $\mathrm{Oh}_{o}=\mathrm{Oh}_{d}=0.05,0.125$, and 0.25 . In the upper row (a)(c), Eo is fixed at 28.8 and the time interval between successive interfaces, $\Delta t_{p}^{*}$, is 2.121. In the lower row (d)-(f), Eo is 144 and $\Delta t_{p}^{*}$ is 0.791 .

formation of a wake. See Fig. 6(b) for further deformation of the drop in (a). The inviscid drop (d), on the other hand, loses fluid to a film pulled off its edge. Since this drop does not develop a vortical wake, it does not form a backwardfacing bag. Similar deformation is also seen for inviscid bubbles. ${ }^{50}$

In the bottom row, the limit of zero surface tension, i.e.,
$\mathrm{Eo}=\infty$ is investigated. Since $\mathrm{Oh}_{o}$ is defined with the surface tension in the denominator, it cannot be used as a measure of viscosity in this case. Instead, another non-dimensional number is defined:

$$
\hat{\mu}=\frac{\mu_{o}}{\sqrt{\rho_{o} \Delta \rho D^{3} a_{z}}}=\frac{1}{\sqrt{\mathrm{Ar}}},
$$

where $\mathrm{Ar}$ is the Archimedes number. Results for three values of $\hat{\mu}=0.01021$ (e), 0.00513 (f), and 0.00204 (g) are compared with results for $\hat{\mu}=0$ (h). In all cases, $\mu_{d} / \mu_{o}=1$. From the plots, it can be seen that since there is no surface tension limiting the deformation, all the drops evolve in a similar way: first an indentation forms at the top, then the drops deform into a forward-facing bag with a thick edge. The effect of $\hat{\mu}$ on the overall shape of the drop is relatively small, with the exception of the rollup of the edge which increases as $\hat{\mu}$ is reduced.

\section{E. Effect of the viscosity ratio}

The results presented so far are all for drops moving in another fluid that has the same or almost the same viscosity. The effect of the viscosity ratio is shown in Fig. 16, where the drops are shown for several values of the governing parameter. In (a) and (b), $\rho_{d} / \rho_{o}=10$ and $\mathrm{Oh}_{o}=0.05$. Eo is 72 in (a) and 144 in (b). In each row, the drop shape is shown at a fixed $t^{*}$ for $\mathrm{Oh}_{d}=0.05,0.125,0.25$, and 1.25 , increasing from left to right. The evolution of the drops in (a) is qualitatively similar for the three lower values of $\mathrm{Oh}_{d}$. They all show a shear breakup mode in which a film of drop liquid is pulled away from the edge of the drop. The drop with the highest $\mathrm{Oh}_{d}$ in the rightmost column, however, has not progressed as far, and will eventually form a forward-facing bag. The comparison in (b), for Eo $=144$, shows the trend observed in (a). Comparisons for drops with $\rho_{d} / \rho_{o}=1.15$ are presented in (c) and (d). In (c), Eo=24 and $\mathrm{Oh}_{o}=0.05$. The drops are shown for $\mathrm{Oh}_{d}=0.0093,0.0466,0.2331$, and 1.1656 (from left to right) at $t^{*}=63.2$. The drops with the three lower viscosity ratios form a backward-facing bag and the drop deformation is most pronounced when the viscosity ratio is smaller. In contrast, the most viscous drop develops a steady disk-like shape. In (d), Eo=144 and $\mathrm{Oh}_{o}=0.25$ and the drops are shown for four different values of the drop Ohnesorge numbers, $\mathrm{Oh}_{d}=0.0093,0.0466,0.2331$, and 1.1656 (from left to right) at $t^{*}=27.1,27.1,46.5$, and 62.0, respectively. The times are not the same because as the viscosity ratio increases, the drops deform much more slowly. Here, the edge of the drop is pulled backward into a thin skirt for the three lower viscosity ratios. The most viscous drop, $\mathrm{Oh}_{d}=1.1656$, does, on the other hand, form a backwardfacing bag.

In Fig. 17, the evolution of the centroid velocity is plotted for the drops shown in Fig. 16. Initially, while the drops are nearly spherical, acceleration is independent of the viscosity of the drop fluid. As the drops start deforming, they slow down due to increased drag. For the drops with $\rho_{d} / \rho_{o}$ $=10$ shown in (a) and (b), the higher viscosity drops deform more slowly and therefore move faster. At a later time, how- 


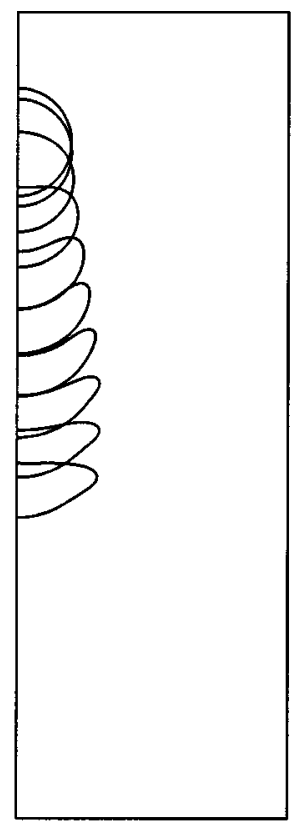

(a) $O h_{o}=0.05$

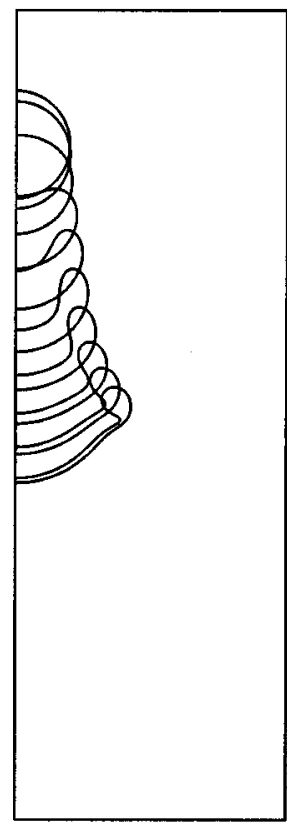

(e) $\hat{\mu}=0.01021$

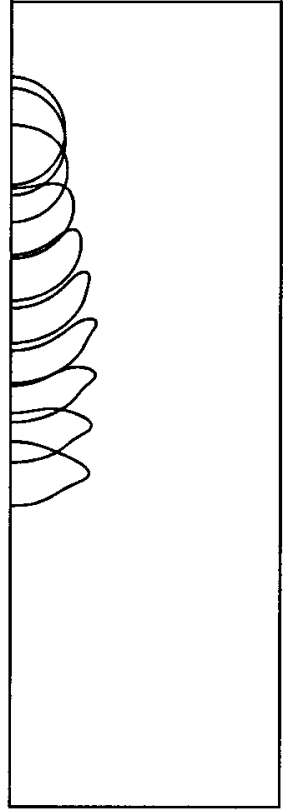

(b) $O h_{o}=0.025$

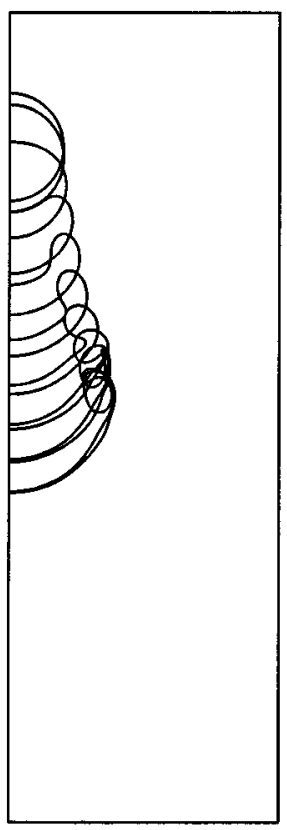

(f) $\hat{\mu}=0.00513$

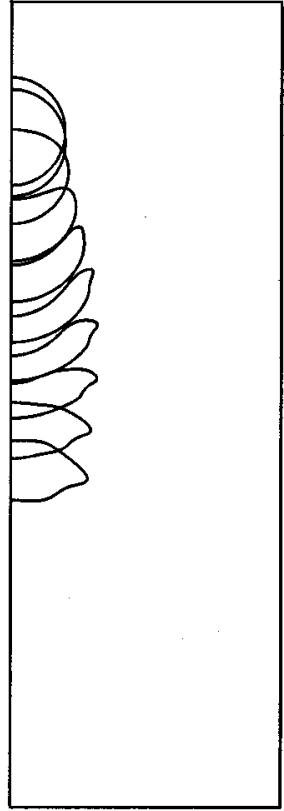

(c) $O h_{o}=0.01$

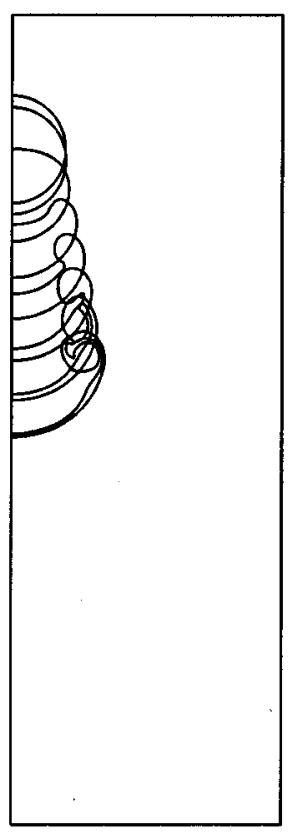

(g) $\hat{\mu}=0.00204$

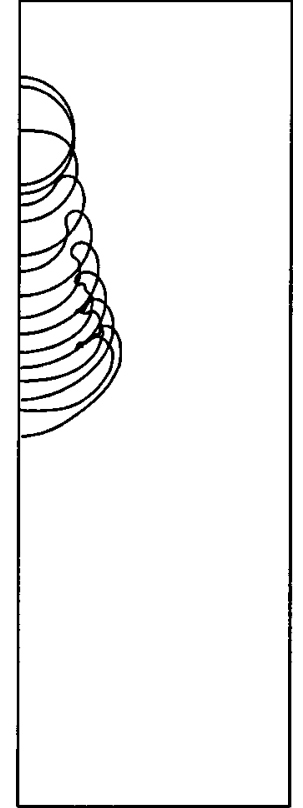

(d) Inviscid

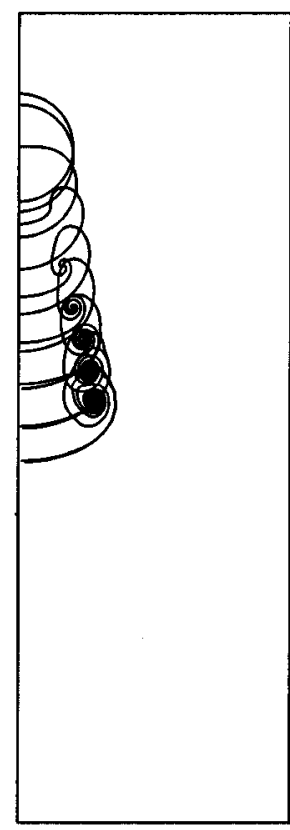

(h) Inviscid

FIG. 15. Effect of $\mathrm{Oh}$ on the initial deformation of drops with $\rho_{d} / \rho_{o}=1.15$. In all cases, $\mu_{d} / \mu_{o}=1$ and the time intervals between successive interfaces, $\Delta t_{p}^{*}=1.581$. In the upper row (a)-(d), Eo=24 and in the lower row (e)-(h), Eo= $=$ (zero surface tension). The viscous simulations (a) $-(\mathrm{c})$ and (e) $-(\mathrm{g})$ were done using a $128 \times 384$ grid. (d) and (h) were done using an inviscid vortex method.

ever, the most viscous drop in (a) forms a backward-facing bag and slows down continually. In (c), where the density ratio is lower, the most viscous drop reaches a steady state shape and velocity. The other drops all form bags and are relatively unaffected by changes in the drop viscosity. In (d), the low viscosity drops speed up again, once a skirt has been pulled off their edges, indicating that the skirt has no significant effects on the motion at this stage. The most viscous drop, on the other hand, forms a backward-facing bag and continues to slow down.
Increasing the drop viscosity reduces its rate of deformation and in some cases, this can result in different breakup modes, changing a shear breakup to a bag breakup, and a bag breakup to a steady-state shape.

\section{F. Deformation and breakup regime maps}

To summarize the results of the various simulations, deformation and breakup maps are presented in Figs. 18 and 19. In the maps, we mark the location of each simulation in 


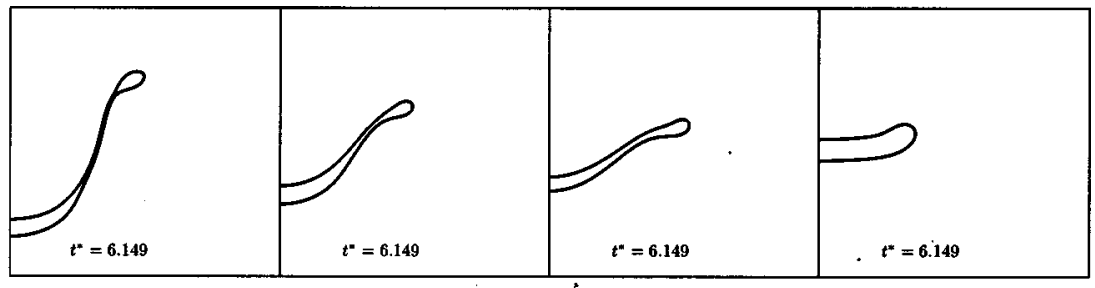

(a) $\rho_{d} / \rho_{o}=10, O h_{o}=0.05, E o=72$

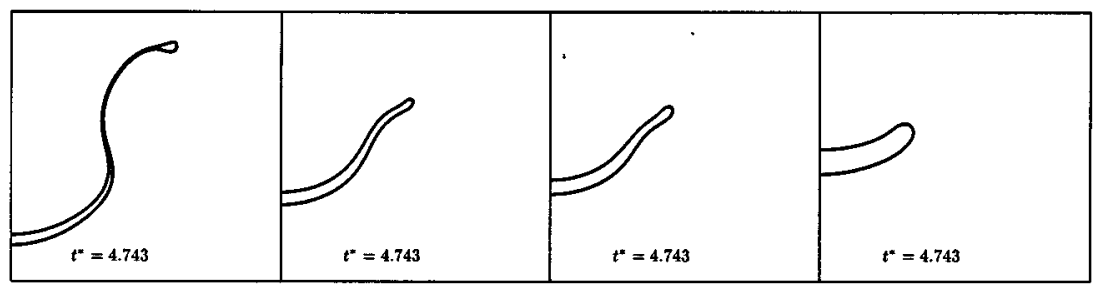

(b) $\rho_{d} / \rho_{o}=10, O h_{o}=0.05, E o=144$

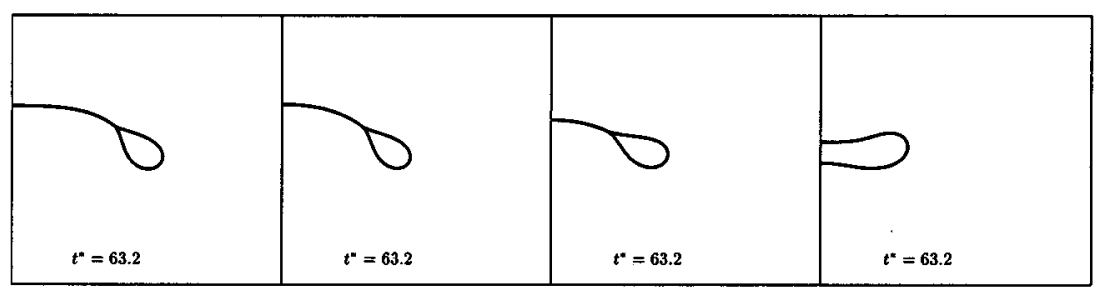

(c) $\rho_{d} / \rho_{o}=1.15, O h_{o}=0.05, E o=24$

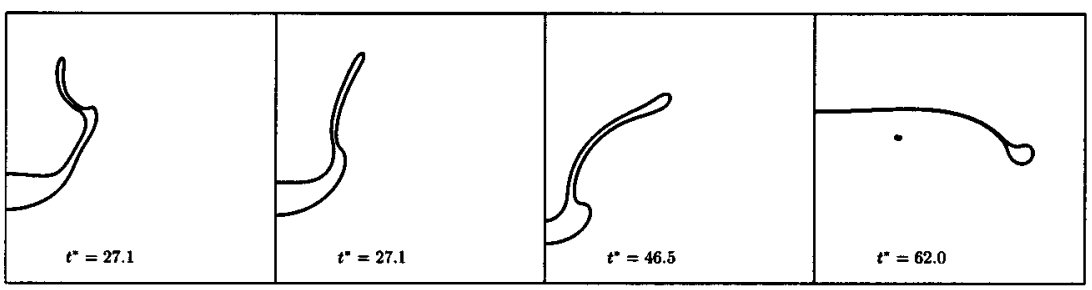

(d) $\rho_{d} / \rho_{o}=1.15, O h_{o}=0.25, E o=144$
FIG. 16. Effect of the viscosity ratio. In each row, the drops at a late time are shown for different values of the drop viscosity while other parameters are fixed. In (a) and (b), $\mathrm{Oh}_{d}=0.05$, $0.125,0.25$, and 1.25 (from left to right). In (c) and (d), $\mathrm{Oh}_{d}=0.0093$, $0.0466,0.2331$, and 1.1656 (from left to right). the $\mathrm{Oh}_{d}$-Eo plane, using a different symbol, depending on the deformation and breakup mode observed. Similar breakup maps have been used to present experimental results by numerous investigators. Figure 18 shows the result of simulations with $\rho_{d} / \rho_{o}=10$. Three maps are shown (a)-(c), corresponding to different ambient Ohnesorge numbers.

The map for a relatively small $\mathrm{Oh}_{o}=0.05$ (a) shows that increasing the magnitude of Eo at a fixed drop Ohnesorge number $\left(\mathrm{Oh}_{d}=0.05\right)$ results in the following transitions between the different breakup modes: oblate ellipsoid $\rightarrow$ backward-facing bag mode $\rightarrow$ transient breakup $\rightarrow$ shear breakup mode. Changing $\mathrm{Oh}_{d}$ for a fixed Eo, on the other hand, yields only minor differences in the breakup mode. Increasing $\mathrm{Oh}_{d}$ from 0.05 to 1.25 , when Eo is fixed at 28.8, for example, does not change the breakup mode. For Eo=72 and 144, a change from a shear breakup mode to a forwardfacing bag mode is observed, as $\mathrm{Oh}_{d}$ is increased from 0.25 to 1.25 . The difference between these two breakup modes is, however, not as significant as that between the backwardfacing bag and the shear breakup modes.

When $\mathrm{Oh}_{o}$ is increased to 0.125 , map (b), the increased viscosity of the surrounding fluid slows the drop down and reduces the rate of deformation. At $\mathrm{Oh}_{d}=0.05$, increasing Eo yields the following transitions between breakup modes: deformed drop $\rightarrow$ backward-facing bag $\rightarrow$ shear breakup. When the boundaries between these breakup modes are compared to the same $\mathrm{Oh}_{d}$ in map (a), some differences are observed. The backward-facing bag, which was observed at $\mathrm{Eo}=28.8$ in (a), is now seen at $\mathrm{Eo}=36$. At $\mathrm{Eo}=48$, the transient breakup is no longer observed and instead we see a backward-facing bag.

The effect of changing $\mathrm{Oh}_{d}$ at a fixed Eo is also examined in (b). When $\mathrm{Eo}=28.8$, changing $\mathrm{Oh}_{d}$ from 0.05 to 1.25 results in only minor differences. The $\mathrm{Oh}_{d}=0.05$ drop displays a prolate shape after an initial oscillatory motion but drops with higher $\mathrm{Oh}_{d}$ deform into oblate ellipsoids with an 


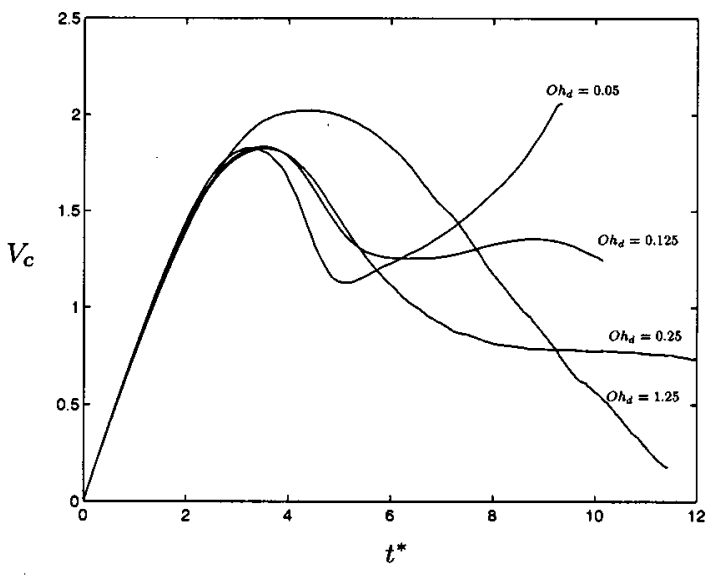

(a) $\rho_{d} / \rho_{o}=10, O h_{o}=0.05, E o=72$

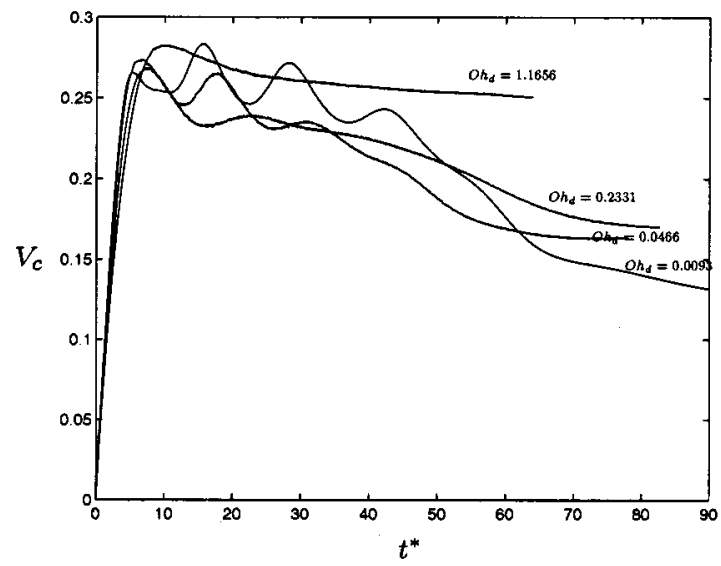

(c) $\rho_{d} / \rho_{o}=1.15, O h_{o}=0.05, E o=24$

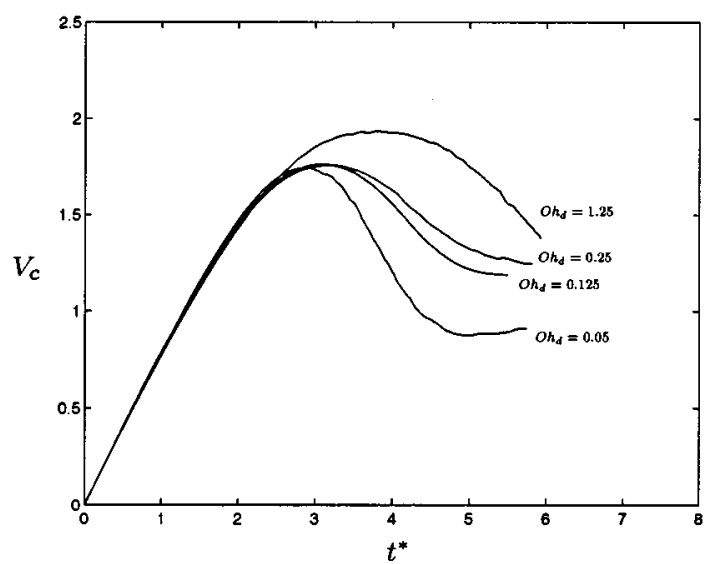

(b) $\rho_{d} / \rho_{o}=10, O h_{o}=0.05, E o=144$

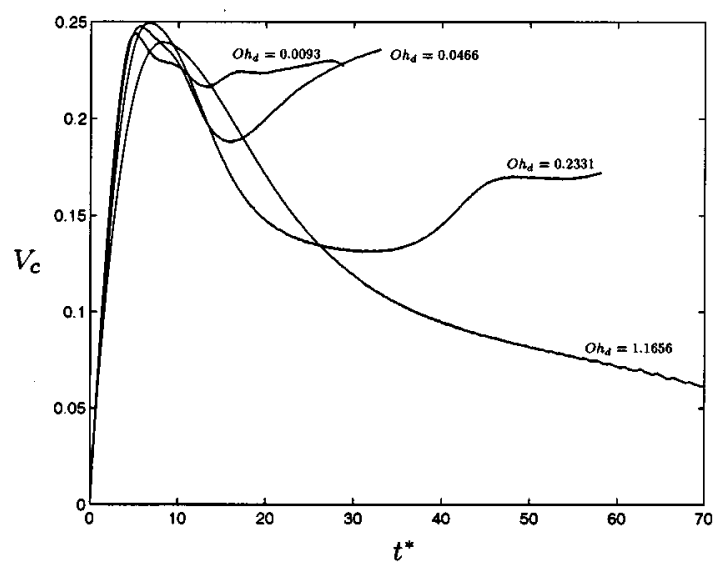

(d) $\rho_{d} / \rho_{o}=1.15, O h_{o}=0.25, E o=144$

FIG. 17. Centroid velocity versus $t^{*}$ for the drops shown in Fig. 16.

indentation (or a dimple) at the top. At $\mathrm{Eo}=72$, the effect of changing $\mathrm{Oh}_{d}$ is more significant. Only the $\mathrm{Oh}_{d}=0.05$ drop shows a shear breakup mode, while the higher $\mathrm{Oh}_{d}$ drops all form a backward-facing bag. At Eo=144, on the other hand, the breakup modes are generally similar to those observed in (a), showing no significant change with $\mathrm{Oh}_{d}$.

When $\mathrm{Oh}_{o}$ is increased to 0.25 in map (c), the effect of the drop viscosity becomes more significant. At $\mathrm{Eo}=28.8$, four simulations with $\mathrm{Oh}_{d}$ ranging from 0.05 to 1.25 show deformed drops, which are similar to those observed in (b) at the same Eo. At Eo=72, four simulations with $\mathrm{Oh}_{d}$ in the same range all show the formation of a backward-facing bag. This is different from the result in (b) for the same Eo, where the shear breakup mode changes to a backward-facing bag mode as $\mathrm{Oh}_{d}$ increases. At $\mathrm{Eo}=144$, only the $\mathrm{Oh}_{d}=0.05$ drop shows the shear breakup mode seen in (a) and (b). The $\mathrm{Oh}_{d}=0.125$ drop deforms into a forward-facing bag and the higher $\mathrm{Oh}_{d}$ drops display a backward-facing bag mode.

In Fig. 19, deformation and breakup regime maps are presented for drops with $\rho_{d} / \rho_{o}=1.15$. Three maps are shown in (a)-(c), for ambient fluids with $\mathrm{Oh}_{o}=0.05,0.25$, and 1.25. The map for $\mathrm{Oh}_{o}=0.05$ (a), displays the following transitions between breakup modes as Eo is increased: oblate ellipsoid $\rightarrow$ backward-facing bag $\rightarrow$ oscillating indented drop $\rightarrow$ forward-facing bag. It is clear that increasing $\mathrm{Oh}_{d}$ has no major effects. The only exception is when $\mathrm{Oh}_{d}$ becomes large $(>1)$ and Eo is relatively low. In this case, the backward-facing bag breakup mode is replaced by a steadily moving indented drop.

When $\mathrm{Oh}_{o}$ is increased to 0.25 (b), a backward-facing bag mode is no longer observed when $\mathrm{Eo}=24$. Instead, a steadily moving indented drop is seen for the $\mathrm{Oh}_{d}$ range investigated. The breakup mode at Eo $=144$ also changes from a forward-facing bag mode to a skirted drop when $\mathrm{Oh}_{d}$ is small $(<1)$. A forward-facing bag mode is observed at $\mathrm{Eo}=288$. As in (a), no noticeable effects of changing $\mathrm{Oh}_{d}$, at a fixed Eo, are observed as long as $\mathrm{Oh}_{d}<1$. When $\mathrm{Oh}_{d}$ $>1$, the drop develops a backward-facing bag when Eo $=144$.

In map (c), $\mathrm{Oh}_{o}=1.25$ and the high viscosity prevents nearly all deformation. When Eo $=24$, the drop remains an oblate ellipsoid but for Eo=144, the drops develop an indentation at the top. The indentation of the more viscous drop 

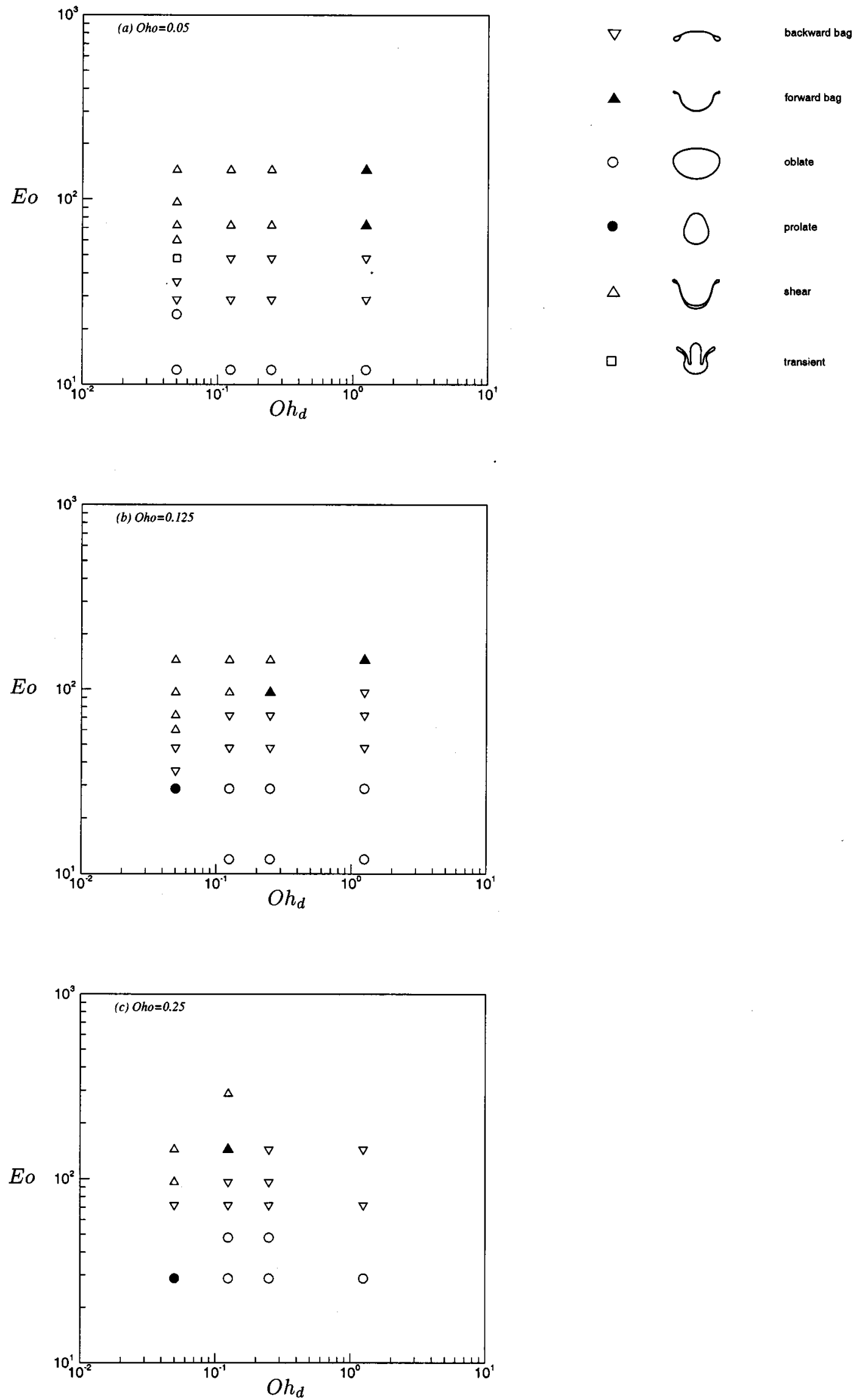

FIG. 18. Deformation and breakup regime maps for $\rho_{d} / \rho_{o}=10$. Three maps are shown for $\mathrm{Oh}_{o}=0.05,0.125$, and 0.25 . In each map, the horizontal and vertical axes are $\mathrm{Oh}_{d}$ and Eo, respectively. 

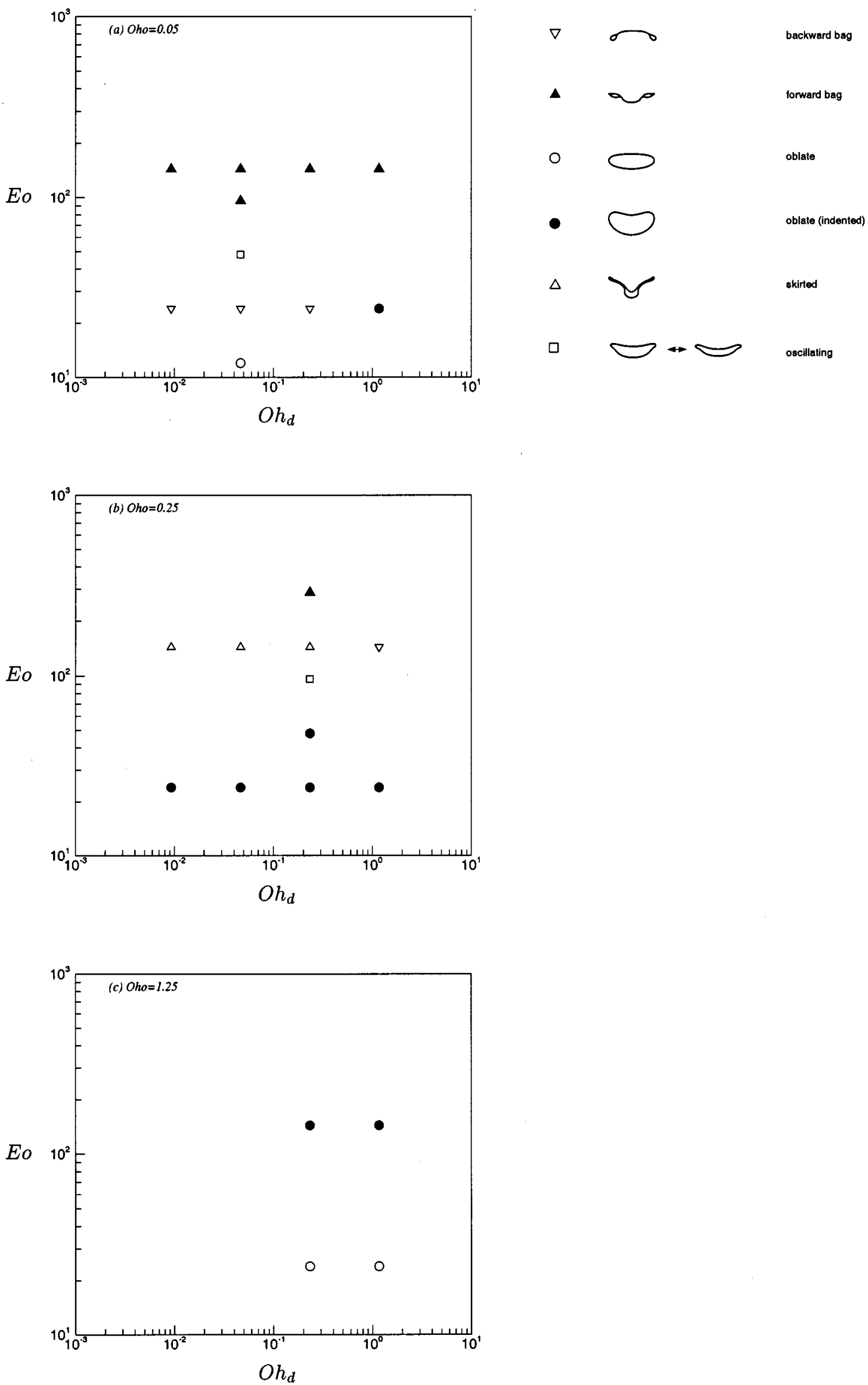

FIG. 19. Deformation and breakup regime maps for $\rho_{d} / \rho_{o}=1.15$. Three maps are shown for $\mathrm{Oh}_{o}=0.05,0.25$, and 1.25 . In each map, the horizontal and vertical axes are $\mathrm{Oh}_{d}$ and Eo, respectively. 
$\left(\mathrm{Oh}_{d}=1.1656\right)$ deepens continuously until it reaches the bottom surface of the drop, forming a vortex ring.

\section{CONCLUSIONS}

The deformation and breakup of axisymmetric drops, accelerated by a constant body force, have been studied by numerical simulations. Results are presented for two density ratios, $\rho_{d} / \rho_{o}=1.15$ and $\rho_{d} / \rho_{o}=10$. For the lower density ratio, the Boussinesq approximation is valid and the results therefore apply for other low density ratios by the simple rescaling discussed in Sec. III. For low Ohnesorge numbers, the Eötvös number and the density ratio are the main controlling parameters. At low density ratios the drop deforms, but does not break up for Eo less than about 18. For 18 $<$ Eo $<36$ (approximately), the drop breaks up by the formation of a backward facing bag. Transient breakup is observed for Eo around 48, and for Eo larger than about 60, the drop evolves into a forward-facing bag.

The formation of a forward-facing bag takes place very quickly (the drop has moved only 3-4 times its initial diameter when the bag is formed) and is essentially an inviscid phenomenon. The formation of a backward-facing bag, on the other hand, takes significantly longer (the drop has moved 8-10 times its initial diameter). A comparison with results obtained by an inviscid vortex method shows that the backward-facing bag is a viscous phenomenon, due to the formation of a low pressure wake behind the drop. Furthermore, the surface area of the drop increases at a faster rate in the forward-facing bag mode.

As $\mathrm{Oh}$ is increased, the effect of the viscosity reduces the rate of deformation. At low Eo, while the drop flattens, its center does not drain completely and a backward-facing bag does not form. As Eo becomes larger, the edges of the drop are pulled outward and sheared off, leading to a "skirted" drop.

When Oh becomes very large, the drop deforms into an oblate ellipsoid at low Eo. At high Eo, baroclinically generated vorticity causes indentation at the top of an accelerated drop, but rapid diffusion of vorticity prevents the roll-up observed for lower Oh. As this indentation continues to grow, the drop finally breaks into a torus. Similar evolution has been seen in simulations of initially oblate drops in Stokes flow (Koh and Leal, ${ }^{31}$ Pozrikidis ${ }^{33}$ ).

The effect of the viscosity ratio is small when $\mathrm{Oh}_{o}$ is small. Although there are differences in the detailed shape of the drop, the overall evolution is generally similar when $\mathrm{Oh}_{d}$ is varied at fixed Eo and $\mathrm{Oh}_{o}$ unless $\mathrm{Oh}_{d}$ is very large. For larger $\mathrm{Oh}_{o}$, the effect of the viscosity ratio becomes more significant and as $\mathrm{Oh}_{d}$ increases, the boundaries between the different breakup modes are moved to higher Eo.

At higher density ratio $\left(\rho_{d} / \rho_{o}=10\right)$ the evolution is similar to the low density ratio case and the effect of the governing parameters is also similar. There are, however, a few important differences. At large Eo, the forward-facing bag seen for the low density case is replaced by a shear breakup mode where the edge of the drop is pulled in the downstream direction, forming a blob of drop fluid connected to the main drop by a thin film. The skirted drop in the low density ratio case is similar in shape (except that no blob was formed). The skirt, however, appears only at a relatively higher $\mathrm{Oh}$ and grows slowly once it has formed. In contrast, the shear breakup mode in the higher density ratio case occurs across the Oh range investigated here. The boundaries between the breakup modes at low Oh remain essentially the same as for the low density ratio case.

In most practical combustion systems, the density difference between the liquid fuel and the high pressure gas is considerably smaller than at atmospheric temperature and pressure. For diesel engines, $\rho_{d} / \rho_{o}=32-53$, for example, (see Heywood ${ }^{51}$ ) and $\rho_{d} / \rho_{o}$ of order unity is common in rocket motors. Nearly all experimental studies of secondary breakup of drops, however, have been done at atmospheric pressures. In the present study we approach the breakup problem from the small density ratio limits, thus complementing previous work. Covering the gap for density ratios between those studied here and the experiments is within the range of present computational capabilities, but requires considerably longer computational times.

\section{ACKNOWLEDGMENTS}

Effort sponsored by the Air Force Office of Scientific Research, Air Force Material Command, USAF, under Grant No. F49620-96-1-0356. The U.S. Government is authorized to reproduce and distribute reprints for Governmental purpose notwithstanding any copyright notation thereon. The views and conclusions contained herein are those of the authors and should not be interpreted as necessarily representing the official policies or endorsements, either expressed or implied, of the Air Force Office of Scientific Research or the U.S. Government. Some of the computations were done using the facilities of the Center of Parallel Computing at the University of Michigan and those at the NAVOCEANO DoD MSRC.

${ }^{1}$ D. A. Drew and S. L. Passman, Theory of Multicomponent Fluids (Springer-Verlag, Berlin, 1998), pp. 135-233.

${ }^{2}$ C. Crowe, M. Sommerfeld, and Y. Tsuji, Multiphase Flows with Droplets and Particles (CRC Press, Boca Raton, 1997), pp. 191-284.

${ }^{3}$ R. D. Reitz and R. Diwakar, "Effect of drop breakup on fuel sprays," SAE Paper No. 860469, 1986.

${ }^{4}$ P. J. O'Rourke and A. A. Amsden, "The TAB method for numerical calculation of spray droplet breakup," SAE Paper No. 872089, 1987.

${ }^{5}$ A. B. Liu, D. Mather, and R. D. Reitz, "Modeling the effects of drop drag and breakup on fuel sprays,', SAE Paper No. 930072, 1993.

${ }^{6}$ A. B. Liu and R. D. Reitz, "Mechanics of air-assisted liquid atomization," Atomization Sprays 3, 55 (1993).

${ }^{7}$ Y. M. Kim and T. S. Wang, "Numerical studies on droplet breakup models," J. Propul. Power 11, 389 (1994).

${ }^{8}$ S.-C. Kong, Z. Han, and R. D. Reitz, “The development and application of a diesel ignition and combustion model for multidimensional engine simulation,"' SAE Paper No. 950278, 1995

${ }^{9}$ G. I. Taylor, "The shape and acceleration of a drop in a high-speed air stream," in The Scientific Papers by G. I. Taylor, edited by G. K. Batchelor (Cambridge University Press, Cambridge, 1963), Vol. 3, pp. 457464.

${ }^{10}$ W. R. Lane, "Shatter of drops in streams of air," Ind. Eng. Chem. 43, 1312 (1951).

${ }^{11}$ J. O. Hinze, "Fundamentals of the hydrodynamic mechanism of splitting in dispersion process," AIChE. J. 1, 289 (1955).

${ }^{12} \mathrm{~F}$. C. Haas, "Stability of droplets suddenly exposed to a high velocity gas stream," AIChE. J. 10, 920 (1964).

${ }^{13}$ A. R. Hanson, E. G. Domich, and H. S. Adams, "Shock tube investigation 
of the breakup of drops by air blasts," Phys. Fluids 6, 1070 (1964).

${ }^{14}$ A. A. Ranger and J. A. Nicholls, "Aerodynamic shattering of liquid drops," AIAA J. 7, 285 (1969).

${ }^{15}$ B. E. Gel'fand, S. A. Gubin, S. M. Kogarko, and S. P. Komar, "Singularities of the breakup of viscous liquid droplets in shock waves," J. Eng. Phys. 25, 1140 (1973).

${ }^{16}$ A. A. Borisov, B. E. Gel'fand, M. S. Natanzon, and O. M. Kossov, "Droplet breakup regimes and criteria for their existence," J. Eng. Phys. 40, 44 (1981).

${ }^{17}$ S. A. Krzeczkowski, "Measurement of liquid droplet disintegration mechanisms,' Int. J. Multiphase Flow 6, 227 (1980).

${ }^{18} \mathrm{M}$. Pilch and C. A. Erdman, "Use of breakup time data and velocity history data to predict the maximum size of stable fragments for acceleration-induced breakup of a liquid drop," Int. J. Multiphase Flow 13, 741 (1987).

${ }^{19} \mathrm{~A}$. Wierzba, "Deformation and breakup of liquid drops in a gas stream at nearly critical Weber numbers," Exp. Fluids 9, 59 (1990).

${ }^{20}$ R. H. Magarvey and B. W. Taylor, "Free fall breakup of large drops," J. Appl. Phys. 27, 1129 (1956).

${ }^{21}$ L.-P. Hsiang and G. M. Faeth, "Near-limit drop deformation and secondary breakup," Int. J. Multiphase Flow 18, 635 (1992).

${ }^{22}$ L.-P. Hsiang and G. M. Faeth, "Drop properties after secondary breakup,' Int. J. Multiphase Flow 19, 721 (1993).

${ }^{23}$ L.-P. Hsiang and G. M. Faeth, "Drop deformation and breakup due to shock wave and steady disturbances," Int. J. Multiphase Flow 21, 545 (1995).

${ }^{24}$ D. D. Joseph, J. Belanger, and G. S. Beavers, "Breakup of a liquid drop suddenly exposed to a high-speed airstream," submitted for publication.

${ }^{25}$ R. Clift, J. R. Grace and M. E. Weber, Bubbles, Drops, and Particles (Academic, New York, 1978), p. 346.

${ }^{26}$ A. H. Lefebvre, Atomization and Sprays (Taylor and Francis, London, 1989), pp. 29-34.

${ }^{27}$ L. Bayvel and Z. Orzechowski, Liquid Atomization (Taylor and Francis, London, 1993), pp. 69-81.

${ }^{28}$ S. S. Sadhal, P. S. Ayyaswamy, and J. N. Chung, Transport Phenomena with Drops and Bubbles (Springer-Verlag, Berlin, 1996), pp. 389-392.

${ }^{29}$ R. I. Nigmatulin, Dynamics of Multiphase Media 1 (Hemisphere, New York, 1991), pp. 150-163.

${ }^{30}$ M. Kojima, E. J. Hinch, and A. Acrivos, "The formation and expansion of a toroidal drop moving in a viscous fluid," Phys. Fluids 27, 19 (1984).

${ }^{31}$ C. J. Koh and L. G. Leal, "The stability of drop shapes for translation at zero Reynolds number through a quiescent fluid," Phys. Fluids A 1, 1309 (1989).

${ }^{32}$ C. J. Koh and L. G. Leal, "An experimental investigation on the stability of viscous drops translating through a quiescent fluid," Phys. Fluids A 2, 2103 (1990)

${ }^{33}$ C. Pozrikidis, "The instability of a moving viscous drop," J. Fluid Mech. 210, 1 (1990).

${ }^{34}$ N. Baumann, D. D. Joseph, P. Mohr, and Y. Renardy, "Vortex rings of one fluid in another in free fall," Phys. Fluids A 4, 567 (1992).

${ }^{35}$ D. S. Dandy and L. G. Leal, "Buoyancy-driven motion of a deformable drop through a quiescent liquid at intermediate Reynolds numbers," J. Fluid Mech. 208, 161 (1989).

${ }^{36} \mathrm{P}$. K. Volkov, "Steady rise of a liquid drop in a viscous fluid," J. Appl. Mech. Tech. Phys. 33, 71 (1992).

${ }^{37}$ L. A. Bozzi, J. Q. Feng, T. C. Scott, and A. J. Pearlstein, "Steady axisymmetric motion of deformable drops falling or rising through a homoviscous fluid in a tube at intermediate Reynolds number,' J. Fluid Mech. 336, 1 (1997).

${ }^{38}$ M. J. Fritts, D. E. Fyre, and E. S. Oran, "Numerical simulations of fuel droplet flows using a Langrangian triangular mesh," NASA CR-168263, 1983

${ }^{39}$ P. Y. Liang, T. W. Eastes, and A. Gharakhari, "Computer simulations of drop deformation and drop breakup," Proceedings of the 24th AIAA/ ASME/SAE/ASEE Joint Propulsion Conference, Boston, MA, AIAA-883142, 1988.

${ }^{40} \mathrm{Z}$. T. Deng and S. M. Jeng, "Numerical simulation of droplet formation in convective flows," AIAA J. 30, 1290 (1992).

${ }^{41}$ Z. T. Deng, G. S. Liaw, and L. Chou, "Modeling of non-spherical droplet dynamics," Proceedings of the 11th Workshop for Computational Fluid Dynamic Applications in Rocket Propulsion, Huntsville, AL, NASA Conference Publication 3221, 1993.

${ }^{42}$ S. P. Seung, C. P. Chen, and Y. S. Chen, "Development of an atomization methodology for spray combustion," in Ref. 41.

${ }^{43}$ S. Zaleski, J. Li, and S. Succi, "Two-dimensional Navier-Stokes simulation of deformation and breakup of liquid patches,' Phys. Rev. Lett. 75, 244 (1995)

${ }^{44}$ S. O. Unverdi and G. Tryggvason, " A front-tracking method for viscous, incompressible, multi-fluid flows," J. Comput. Phys. 100, 25 (1992).

${ }^{45}$ F. H. Harlow and J. E. Welch, "Numerical calculation of time-dependent viscous incompressible flow of fluid with free surface," Phys. Fluids 8, 2182 (1965).

${ }^{46} \mathrm{G}$. Tryggvason, B. Bunner, O. Ebrat, and W. Tauber, "Computations of multiphase flows by a finite difference/front tracking method-I. Multifluid flows,'29th Computational Fluid Dynamics, von Karman Institute for Fluid Dynamics Lecture Series 1998-03, 1998.

${ }^{47}$ W. J. A. Dahm, C. M. Scheil, and G. Tryggvason, "Dynamics of vortex interaction with a density interface," J. Fluid Mech. 205, 1 (1989).

${ }^{48} \mathrm{~T}$. Wairegi and J. R. Grace, "The behavior of large drops in immiscible liquids,' Int. J. Multiphase Flow 3, 67 (1976).

${ }^{49}$ W. J. A. Dahm, C. E. Frieler, and G. Tryggvason, "Vortex structure and dynamics in the near field of a coaxial jet," J. Fluid Mech. 241, 371 (1992).

${ }^{50} \mathrm{G}$. R. Baker and D. W. Moore, "The rise and distortion of a twodimensional gas bubble in an inviscid liquid," Phys. Fluids A 1, 1451 (1989).

${ }^{51}$ J. B. Heywood, Internal Combustion Engine Fundamentals (McGrawHill, New York, 1988), p. 522. 